\title{
Boosted Electronic, NLO and Absorption Characteristics for Quercetin and Taxifolin: Comparative Experimental and DFT Studies
}

\author{
Mohamed Ahmed Mohamed El-Mansy 1,2,*iD, Athisaya Rajah Suvitha ${ }^{3(D)}$, \\ Nookala Yuvaraj Maharani ${ }^{4}$ (D), Agalya Vedi 5 (D) \\ 1 Molecular Modeling Simulation Lab., Department of Physics, Faculty of Education, Ain Shams University, Roxy, Cairo, \\ Egypt \\ 2 Condensed Matter Theory Group, Department of Physics, College of Science and Arts, Qassim University, Ar Rass, 51921, \\ Saudi Arabia \\ 3 Department of Physics, CMR Institute of Technology, Bengaluru, Karnataka, India \\ 4 Department of Physics, Gopalan College of Engineering, Bengaluru, Karnataka, India \\ 5 Department of EEE, CMR Institute of Technology, Bengaluru, Karnataka, India \\ * Correspondence: Profmelmansy@gmail.com;
}

Received: 17.10.2021; Revised: 17.11.2021; Accepted: 19.11.2021; Published: 2.12.2021

\begin{abstract}
In the present study, a considerable, reproducible, and eco-friendly biological synthesis of $\mathrm{Ag}$ nanoparticles using Mangifera indica leaf extract as a reductant is documented. The spectroscopic characteristics of synthesized Ag nanoparticles are described by both UV-Vis and FT-IR techniques. The bandgap offsets, reactivity, and NLO properties for two flavonoids, quercetin, and taxifolin, are examined using the DFT approach. Also, a detailed comparative analysis for HOMO-LUMO interactions among quercetin and taxifolin is discussed. Results show that quercetin and taxifolin possess dipole moment (DM=4.79, 3.99 Debye) and bandgap offset $(2.59,2.98 \mathrm{eV})$. Both molecules are promising candidates as window layers for solar cells and memory switch devices. In addition, hyperpolarizability calculations show that quercetin NLO response is higher than taxifolin, which sets a revolutionary recall for NLO manufacture upgrade. Moreover, NBO and UV-Vis absorption characteristics are reported as well.
\end{abstract}

Keywords: Ag nanoparticles extract; quercetin and taxifolin; NBO; NLO; HOMO-LUMO; MEP; DFT.

(C) 2021 by the authors. This article is an open-access article distributed under the terms and conditions of the Creative Commons Attribution (CC BY) license (https://creativecommons.org/licenses/by/4.0/).

\section{Introduction}

Recently, Ag nanoparticles synthesis has been developed efficiently owing to their outstanding applications in both biological, optical, and nonlinear optical manufactured devices [1-3]. Pristine Ag nanoparticles have the lowest fatigue resistance as well as the highest electrical and thermal conductivity among other metals [4-7]. There are different studies of the use of green nanomaterial synthesis agents, i.e., natural and environmentally friendly reducing and capping agents [8-10]. In several plants and related plant products, an entire class of flavonoids is present in abundance. Five flavonoids, specifically (-)- epicatechin-3-O- $\beta$-glucopyranoside, 5-hydroxy/3 (4-hydroxylphenyl) pyrano [3,2-g]chromene/4(8H)one, 6(phydroxybenzyl) taxifolin/7O/ $\beta$-D-glucoside, quercetin-3-O- $\alpha$-glucopyranosyl- $(1 \rightarrow 2)-\beta$-Dgluco-pyranoside $\&(-)-$ epicatechin(2(3,4dihydroxyphenyl)3,4dihydro(2H)chromene(3,5,7)triol were detached from 
mango leaves (Mangifera indica L.). Quercetin (QCN) and Taxifolin (TXN) compositions are contained in Mangifera indica extracts with polyphenolic compounds. Antifungal activities of both QCN and TXN have shown an increase in their antifungal behaviors upon concentration rise [11-13]. QCN is an efficient scavenging antioxidant, although that activity relays firmly upon the intracellular reduced glutathione availability [14-16]. Also, QCN possesses a right, pro-apoptotic influence in cellular tumors and prohibits human cancer cell growth at diversional phases of the cell cycle [17-19], which in turn act as chemo-preventers [20-23]. On the other hand, TXN prevented skin scar cell carcinoma growth [24] and was promoted as myeloperoxidase (MPO)-derived RNS scavenger [25,26]. Moreover, TXN decreases BSOinduced injury to dermal fibroblasts as well as downregulates collagenase I (MMP-1) in UVBtreated skin cells. Up to our knowledge, only a few reports have been revealed on $\mathrm{Ag}$ nanoparticles synthesis using pure flavonoid reduction. Accordingly, the present study uncovers both synthesis, spectroscopic and quantum chemical investigations for QCN and TXN compounds. In addition, electronic, optical, and nonlinear optical (NLO) characterization behaviors are studied to foresee the desired applications for QCN and TXN.

\section{Materials and Methods}

\subsection{Extraction of Ag nanoparticles.}

Fresh Mangifera Indica leaves are picked up from Bangalore spot, Karnataka, India. The collected leaves are washed a few times under running faucet water followed by twofold refined water (DDM) to clear any residuals or dampness. The washed Mango leaves are left to dry well, and then a sliced $10 \mathrm{~g}$ of leaves are bubbled in a hot water shower $\left(\sim 80^{\circ} \mathrm{C}\right)$ inside 500 $\mathrm{ml}$ Erlenmeyer cup along in $100 \mathrm{ml}$ sterile DDW for 30 mins. Then, the concentrated solution is cooled and filtrated well to maintain $\mathrm{AgNO}_{3}$ nanoparticles. Distinctive $10 \mathrm{ml}$ mango leaves extracted, added to $30 \mathrm{ml} \mathrm{AgNO}_{3}$, and stirred for $1 \mathrm{~h}$ to turn $\mathrm{Ag}^{+}$into $\mathrm{Ag}$. The development of Ag nanoparticles is confirmed by turning stirred mixture contrast into ruddy earth color. After that, the stirred mixture is centrifuged at $2000 \mathrm{rpm}$ for 30 mins. The supernatant fluid is disposed of, and the gooey Ag remainder is collected over Whatman No. 1 paper and left for $48 \mathrm{~h}$. Finally, the dried Ag nanoparticles are assembled and moved to light-safe compartments.

\subsection{FT-IR and UV-Vis investigations.}

FT-IR chart is measured 4000-400 $\mathrm{cm}^{-1}$ through the BRUKER IFS- 66V FT-IR instrument with $\mathrm{KBr}$ pellet style. Also, the UV-Vis chart is scanned in region $200-700 \mathrm{~nm}$ utilizing a twofold pillar spectrophotometer (Metash UV-Vis, model UV-8500).

\subsection{Computational details.}

DFT calculations of QCN and TXN are achieved as built-in G09 [27] and visualized by GV5 [28]. The well-examined WB97XD [29-37] is employed due to the premium electronic representation revealed for various structural systems [38-48]. The simulated QCN and TXN are optimized and shown in Figure 1. The optimal geometries for both QCN and TXN are collected in Table 1. A comparison between measured and computed wavenumbers with corresponding assignments for both QCN and TXN is shown in Table 2. All calculated wavenumbers are multiplied by a factor $(\sim 0.98)$ to concur with the experimental results. Both Experimental and simulated IR spectra for both QCN and TXN are displayed in Figure 2. Also, 
nonlinear optical (NLO) parameters as static-polarizability $(\alpha)$ and $1^{\text {st }}$ order hyperpolarizability $(\beta)$ for both QCN and TXN are computed and registered in Tables 3. Comparative physical indices such as EHOMO, ELUMO, HOMO/LUMO offset, spin, ionizing-potential (I), electronic-affinity $(\mathrm{A})$, chemical-potential $(\chi)$, hardness $(\kappa)$, electrophile-index $(\phi)$, softness (s), total dipole-moment (TDM), static polarizability $\alpha_{\text {tot }}$, polarizability anisotropy $\Delta \alpha$ and first-order hyperpolarizability $\beta_{\text {tot }}$ for QCN and TXN with other similar published compounds are calculated and collected in Table 4. Finally, natural bond orbital (NBO) and UV-Vis absorption analyses have been carried out B3LYP/6-311++(G,p). The $2^{\text {nd }}$ order Fock matrix is performed to speculate acceptor-donor interplays in NBO. For each donor $(i) \&$ acceptor $(j)$, the stabilization-energy E (2) correlating with the delocalization $i \rightarrow j$ is given as:

$$
E^{(2)}=\Delta \mathrm{E}_{i j}=\frac{q_{i} F(\mathrm{i}, \mathrm{j})^{2}}{\varepsilon_{i-} \varepsilon_{j}}
$$

where $q_{i}$ is the donor orbital occupancy, $\varepsilon_{i} \& \varepsilon_{j}$ are diagonal_elements, and $F(i, j)$ is the NBO/off-diagonal Fock matrix.

\section{Results and Discussion}

\subsection{Structural and FT-IR analyses.}

The optimum geometries for both QCN and TXN are achieved by WB97XD/Lan2MB and SDD Levels shown in Figure 1 and Table 1. From Table 1 and Figure 1, we may clarify the following:

The optimal bond length for $\mathrm{C}=\mathrm{C}$ is in the range 1.40 to $1.42 \AA$ that compares well with experimental data $1.48 \AA$ [49]. The $\mathrm{C}=\mathrm{O}$ bond length is somewhat longer than experimental data $1.23 \AA$ [49] for QCN and TXN. Also, the computed bond extent for all O-H terminals is similar $1.02 \AA$. The calculations show that stable structures for both QCN and TXN are twisted via hydroxy-phenol moiety with dihedral angles $\mathrm{C} 12 \mathrm{C} 10 \mathrm{C} 11 \mathrm{C} 17=133.35^{\circ}$ and C9C8C10C16 $=-102.07^{\circ}$, respectively. QCN is expected to be more active than TXN due to $\pi$-electrons resonance along with nearby withdrawing groups. TXN has two extra $\mathrm{H}$ antennae over QCN, which holds $\pi$-electrons in aromatic moiety lowering its dipole moment.

Calculated IR spectra for both QCN and TXN have been achieved using WB97XD/SDD. Both experimental, computed IR wavenumbers, IR intensity, and desired assignments are collected in Table 2 and presented in Fig. 2. Detailed assignments are briefly discussed as the following:

The $\mathrm{OH}$ stretch is monitored in the range $3500-3200 \mathrm{~cm}^{-1}$ [50]. Mode 1 is assigned to $\mathrm{OH}$ stretch at $3262(\mathrm{QCN}), 3386(\mathrm{TXN}) \mathrm{cm}^{-1}$, which is comparable to the experimental peak $3416 \mathrm{~cm}^{-1}$. Mode 6 is attributed to $\mathrm{OH}$ inplane bending at $1415(\mathrm{QCN}), 1404(\mathrm{TXN}) \mathrm{cm}^{-1}$ corresponds to measured value $1419 \mathrm{~cm}^{-1}$. Asymmetric and symmetric $\mathrm{CH}$ stretch are tracked at $3200-2800 \mathrm{~cm}^{-1}$ [51]. Modes $2 \& 3$ are corresponding to both Asymmetric and Symmetric $\mathrm{CH}$ stretch at 3191; $3168(\mathrm{QCN}), 3040 ; 2998(\mathrm{TXN}) \mathrm{cm}^{-1}$, respectively; match with indexed data at 2920 and $2851 \mathrm{~cm}^{-1}$. Modes $7 \& 8$ are recognized as $\mathrm{C}-\mathrm{H}$ inplane bending at 1378-1273 (QCN), 1387-1284 (TXN) $\mathrm{cm}^{-1}$, respectively; that approved with found peaks at 1363-1273 $\mathrm{cm}^{-1}$. Mode 10 is specified for both $\mathrm{OH}$ and $\mathrm{CH}$ out plane bending at $594(\mathrm{QCN})$ and 617 $(\mathrm{TXN}) \mathrm{cm}-1$, corresponding to tracked peak at $608 \mathrm{~cm}^{-1}$. The $\mathrm{C}=\mathrm{C}$ stretch is detected in domain $1800-1600 \mathrm{~cm}^{-1}[52,53]$. Modes $4 \& 5$ are assigned to $\mathrm{C}=\mathrm{C}$ stretch at $1705-1658(\mathrm{QCN}), 1692-$ $1628(\mathrm{TXN}) \mathrm{cm}^{-1}$, respectively; that agreed with experimental data at $1823-1608 \mathrm{~cm}^{-1}$. Mode 9 
is identified for C-O+C-C stretch at $882(\mathrm{QCN}), 889(\mathrm{TXN}) \mathrm{cm}^{-1}$, concord with an indexed peak at $890 \mathrm{~cm}^{-1}$. Our Calculations have shown a good agreement with measured data, especially with QCN molecules. It is convenient to relate any deviations between computed results (single molecule-gas phase) and experimental frequencies (metamolecules-solid phase) to extended hydrogens and packing interactions.
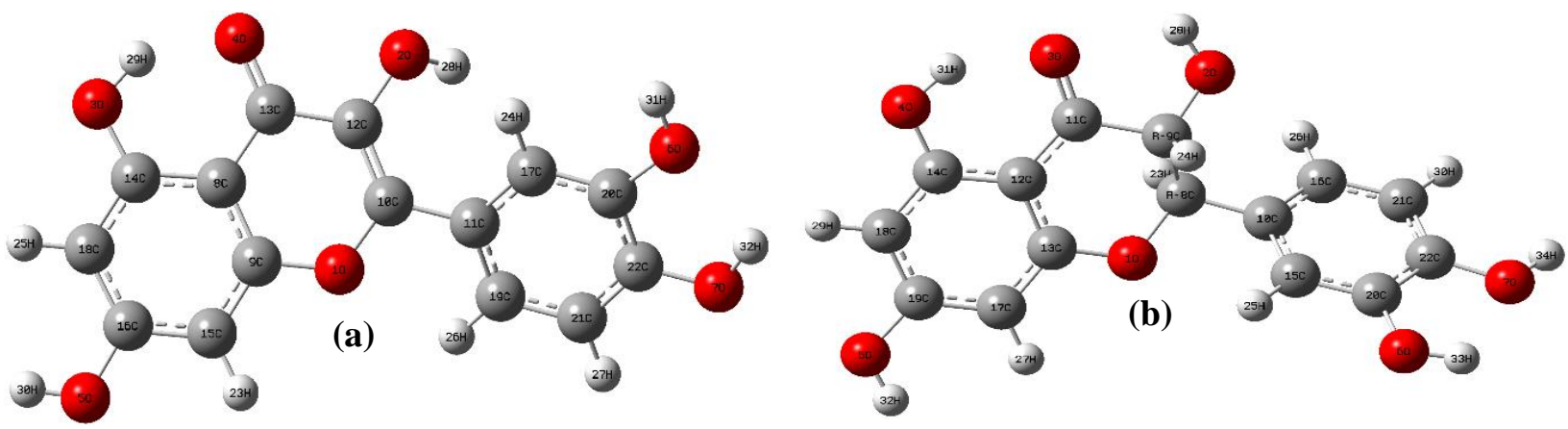

Figure 1. Optimized structure and numbering scheme for (a) QCN, (b) TXN at WB97XD/SDD.

Table 1. Structural parameters for both QCN and TXN at WB97XD aided with Lanl2MB and SDD sets.

\begin{tabular}{|c|c|c|c|c|c|}
\hline & \multicolumn{2}{|c|}{ QCN } & & \multicolumn{2}{|c|}{ TXN } \\
\hline & Lanl2MB & SDD & & Lanl2MB & SDD \\
\hline Bond Length $(\AA)$ & & & Bond Length (Å) & & \\
\hline O1-C9 & 1.41 & 1.41 & $\mathrm{O} 1-\mathrm{C} 8$ & 1.49 & 1.49 \\
\hline O1-C10 & 1.41 & 1.41 & $\mathrm{O} 1-\mathrm{C} 13$ & 1.41 & 1.41 \\
\hline $\mathrm{O} 2-\mathrm{C} 12$ & 1.41 & 1.41 & $\mathrm{O} 2-\mathrm{C} 9$ & 1.45 & 1.45 \\
\hline $\mathrm{O} 2-\mathrm{H} 28$ & 1.02 & 1.02 & $\mathrm{O} 2-\mathrm{H} 28$ & 1.03 & 1.03 \\
\hline $\mathrm{O} 3-\mathrm{C} 14$ & 1.36 & 1.36 & $\mathrm{O} 3-\mathrm{C} 11$ & 1.29 & 1.29 \\
\hline $\mathrm{O} 3-\mathrm{H} 29$ & 1.10 & 1.10 & $\mathrm{O} 4-\mathrm{C} 14$ & 1.37 & 1.37 \\
\hline O4-C13 & 1.30 & 1.30 & O4-H31 & 1.07 & 1.07 \\
\hline O5-C16 & 1.40 & 1.40 & O5-C19 & 1.40 & 1.40 \\
\hline O5-H30 & 1.02 & 1.02 & O5-H32 & 1.02 & 1.02 \\
\hline O6-C20 & 1.42 & 1.42 & O6-C20 & 1.40 & 1.40 \\
\hline O6-H31 & 1.02 & 1.02 & O6-H33 & 1.02 & 1.02 \\
\hline $\mathrm{O} 7-\mathrm{C} 22$ & 1.41 & 1.41 & $\mathrm{O} 7-\mathrm{C} 22$ & 1.41 & 1.41 \\
\hline O7-H32 & 1.02 & 1.02 & O7-H34 & 1.01 & 1.01 \\
\hline C8-C9 & 1.42 & 1.42 & C8-C9 & 1.58 & 1.58 \\
\hline C8-C13 & 1.46 & 1.46 & C8-C10 & 1.54 & 1.54 \\
\hline C8-C14 & 1.45 & 1.45 & $\mathrm{C} 8-\mathrm{H} 23$ & 1.11 & 1.11 \\
\hline C9-C15 & 1.40 & 1.40 & C9-C11 & 1.55 & 1.55 \\
\hline C10-C111 & 1.51 & 1.51 & C9-H24 & 1.12 & 1.12 \\
\hline C10-C12 & 1.38 & 1.38 & C10-C15 & 1.41 & 1.41 \\
\hline C11-C17 & 1.41 & 1.41 & $\mathrm{C} 10-\mathrm{C} 16$ & 1.40 & 1.40 \\
\hline C11-C19 & 1.41 & 1.41 & C11-C12 & 1.44 & 1.44 \\
\hline C12-C13 & 1.49 & 1.49 & C12-C13 & 1.43 & 1.43 \\
\hline C14-C18 & 1.42 & 1.42 & $\mathrm{C} 12-\mathrm{C} 14$ & 1.44 & 1.44 \\
\hline $\mathrm{C} 15-\mathrm{C} 16$ & 1.42 & 1.42 & C13-C17 & 1.40 & 1.40 \\
\hline $\mathrm{C} 15-\mathrm{H} 23$ & 1.09 & 1.09 & C14-C18 & 1.42 & 1.42 \\
\hline C16-C18 & 1.41 & 1.41 & $\mathrm{C} 15-\mathrm{C} 20$ & 1.40 & 1.40 \\
\hline $\mathrm{C} 17-\mathrm{C} 20$ & 1.41 & 1.41 & $\mathrm{C} 15-\mathrm{H} 25$ & 1.09 & 1.09 \\
\hline C17-H24 & 1.10 & 1.10 & C16-C21 & 1.41 & 1.41 \\
\hline $\mathrm{C} 18-\mathrm{H} 25$ & 1.09 & 1.09 & $\mathrm{C} 16-\mathrm{H} 26$ & 1.10 & 1.10 \\
\hline C19-C21 & 1.40 & 1.40 & C17-C19 & 1.43 & 1.43 \\
\hline $\mathrm{C} 19-\mathrm{H} 26$ & 1.10 & 1.10 & $\mathrm{C} 17-\mathrm{H} 27$ & 1.09 & 1.09 \\
\hline $\mathrm{C} 20-\mathrm{C} 22$ & 1.43 & 1.43 & C18-C19 & 1.41 & 1.41 \\
\hline C21-C22 & 1.41 & 1.41 & C18-H29 & 1.09 & 1.09 \\
\hline \multirow[t]{3}{*}{$\mathrm{C} 21-\mathrm{H} 27$} & 1.10 & 1.10 & $\mathrm{C} 20-\mathrm{C} 22$ & 1.43 & 1.43 \\
\hline & & & C21-C22 & 1.40 & 1.40 \\
\hline & & & C21-H30 & 1.10 & 1.10 \\
\hline Bond Angle ( $\left.{ }^{\circ}\right)$ & & & Bond Angle $\left({ }^{\circ}\right)$ & & \\
\hline C9-O1-C10 & 116.22 & 116.22 & $\mathrm{C} 8-\mathrm{O} 1-\mathrm{C} 13$ & 112.35 & 112.35 \\
\hline $\mathrm{C} 12-\mathrm{O} 2-\mathrm{H} 28$ & 102.87 & 102.87 & $\mathrm{C} 9-\mathrm{O} 2-\mathrm{H} 28$ & 99.09 & 99.09 \\
\hline C14-03-H29 & 101.26 & 101.26 & C14-O4-H31 & 101.96 & 101.96 \\
\hline C16-O5-H30 & 103.22 & 103.22 & C19-O5-H32 & 103.43 & 103.43 \\
\hline C20-O6-H31 & 103.58 & 103.58 & C20-O6-H33 & 100.47 & 100.47 \\
\hline
\end{tabular}




\begin{tabular}{|c|c|c|c|c|c|}
\hline & \multicolumn{2}{|c|}{ QCN } & & \multicolumn{2}{|c|}{ TXN } \\
\hline & Lanl2MB & SDD & & Lanl2MB & SDD \\
\hline C22-O7-H32 & 103.60 & 103.60 & C22-O7-H34 & 104.41 & 104.41 \\
\hline C9-C8-C13 & 122.20 & 122.20 & O1-C8-C9 & 111.96 & 111.96 \\
\hline C9-C8-C14 & 120.18 & 120.18 & O1-C8-C10 & 107.00 & 107.00 \\
\hline C13-C8-C14 & 117.60 & 117.60 & O1-C8-H23 & 109.12 & 109.12 \\
\hline O1-C9-C8 & 121.88 & 121.88 & C9-C8-C10 & 111.42 & 111.42 \\
\hline O1-C9-C15 & 116.93 & 116.93 & C9-C8-H23 & 107.84 & 107.84 \\
\hline C8-C9-C15 & 121.20 & 121.20 & $\mathrm{C} 10-\mathrm{C} 8-\mathrm{H} 23$ & 109.47 & 109.47 \\
\hline O1-C10-C11 & 111.00 & 111.00 & $\mathrm{O} 2-\mathrm{C} 9-\mathrm{C} 8$ & 111.75 & 111.75 \\
\hline $\mathrm{O} 1-\mathrm{C} 10-\mathrm{C} 12$ & 125.31 & 125.31 & O2-C9-C11 & 109.49 & 109.49 \\
\hline C11-C10-C12 & 123.65 & 123.65 & O2-C9-H24 & 111.91 & 111.91 \\
\hline C10-C11-C17 & 119.60 & 119.60 & C8-C9-C11 & 107.21 & 107.21 \\
\hline C10-C11-C19 & 121.21 & 121.21 & C8-C9-H24 & 107.90 & 107.90 \\
\hline C17-C11-C19 & 119.19 & 119.19 & C11-C9-H24 & 108.41 & 108.41 \\
\hline $\mathrm{O} 2-\mathrm{C} 12-\mathrm{C} 10$ & 124.78 & 124.78 & C8-C10-C15 & 119.65 & 119.65 \\
\hline $\mathrm{O} 2-\mathrm{C} 12-\mathrm{C} 13$ & 115.50 & 115.50 & $\mathrm{C} 8-\mathrm{C} 10-\mathrm{C} 16$ & 120.04 & 120.04 \\
\hline C10-C12-C13 & 119.71 & 119.71 & C15-C10-C16 & 120.29 & 120.29 \\
\hline $\mathrm{O} 4-\mathrm{C} 13-\mathrm{C} 8$ & 121.06 & 121.06 & O3-C11-C9 & 119.31 & 119.31 \\
\hline O4-C13-C12 & 124.32 & 124.32 & $\mathrm{O} 3-\mathrm{C} 11-\mathrm{C} 12$ & 123.33 & 123.33 \\
\hline C8-C13-C12 & 114.62 & 114.62 & C9-C11-C12 & 117.35 & 117.35 \\
\hline O3-C14-C8 & 118.92 & 118.92 & C11-C12-C13 & 121.27 & 121.27 \\
\hline O3-C14-C18 & 122.58 & 122.58 & C11-C12-C14 & 117.66 & 117.66 \\
\hline C8-C14-C18 & 118.50 & 118.50 & $\mathrm{C} 13-\mathrm{C} 12-\mathrm{C} 14$ & 121.01 & 121.01 \\
\hline C9-C15-C16 & 118.24 & 118.24 & $\mathrm{O} 1-\mathrm{C} 13-\mathrm{C} 12$ & 122.84 & 122.84 \\
\hline C9-C15-H23 & 121.24 & 121.24 & O1-C13-C17 & 117.67 & 117.67 \\
\hline $\mathrm{C} 16-\mathrm{C} 15-\mathrm{H} 23$ & 120.53 & 120.53 & C12-C13-C17 & 119.47 & 119.47 \\
\hline O5-C16-C15 & 115.72 & 115.72 & $\mathrm{O} 4-\mathrm{C} 14-\mathrm{C} 12$ & 119.00 & 119.00 \\
\hline $\mathrm{O} 5-\mathrm{C} 16-\mathrm{C} 18$ & 122.08 & 122.08 & $\mathrm{O} 4-\mathrm{C} 14-\mathrm{C} 18$ & 122.03 & 122.03 \\
\hline C15-C16-C18 & 122.20 & 122.20 & C12-C14-C18 & 118.97 & 118.97 \\
\hline C11-C17-C20 & 120.93 & 120.93 & $\mathrm{C} 10-\mathrm{C} 15-\mathrm{C} 20$ & 119.74 & 119.74 \\
\hline C11-C17-H24 & 120.29 & 120.29 & $\mathrm{C} 10-\mathrm{C} 15-\mathrm{H} 25$ & 120.64 & 120.64 \\
\hline $\mathrm{C} 20-\mathrm{C} 17-\mathrm{H} 24$ & 118.78 & 118.78 & $\mathrm{C} 20-\mathrm{C} 15-\mathrm{H} 25$ & 119.62 & 119.62 \\
\hline C14-C18-C16 & 119.68 & 119.68 & $\mathrm{C} 10-\mathrm{C} 16-\mathrm{C} 21$ & 120.65 & 120.65 \\
\hline $\mathrm{C} 14-\mathrm{C} 18-\mathrm{H} 25$ & 119.77 & 119.77 & C10-C16-CH26 & 119.83 & 119.83 \\
\hline C16-C18-H25 & 120.55 & 120.55 & C21-C16-H26 & 119.52 & 119.52 \\
\hline C11-C19-C21 & 120.53 & 120.53 & C13-C17-C19 & 119.18 & 119.18 \\
\hline C11-C19-H26 & 120.17 & 120.17 & C13-C17-H27 & 120.21 & 120.21 \\
\hline C21-C19-H26 & 119.23 & 119.23 & C19-C17-H27 & 120.60 & 120.60 \\
\hline C6-C20-C17 & 117.71 & 117.71 & C14-C18-C19 & 118.96 & 118.96 \\
\hline C6-C20-C22 & 122.81 & 122.81 & $\mathrm{C} 14-\mathrm{C} 18-\mathrm{H} 29$ & 120.82 & 120.82 \\
\hline $\mathrm{C} 17-\mathrm{C} 20-\mathrm{C} 22$ & 119.44 & 119.44 & $\mathrm{C} 19-\mathrm{C} 18-\mathrm{H} 29$ & 120.22 & 120.22 \\
\hline C19-C21-C22 & 120.67 & 120.67 & O5-C19-C17 & 121.22 & 121.22 \\
\hline C19-C21-H27 & 120.69 & 120.69 & O5-C19-C18 & 116.42 & 116.42 \\
\hline $\mathrm{C} 22-\mathrm{C} 21-\mathrm{H} 27$ & 118.62 & 118.62 & C17-C19-C18 & 122.36 & 122.36 \\
\hline $\mathrm{O} 7-\mathrm{C} 22-\mathrm{C} 20$ & 122.92 & 122.92 & O6-C20-C15 & 121.72 & 121.72 \\
\hline $\mathrm{O} 7-\mathrm{C} 22-\mathrm{C} 21$ & 117.86 & 117.86 & O6-C20-C22 & 118.77 & 118.77 \\
\hline \multirow[t]{7}{*}{$\mathrm{C} 20-\mathrm{C} 22-\mathrm{C} 21$} & 119.19 & 119.19 & $\mathrm{C} 15-\mathrm{C} 20-\mathrm{C} 22$ & 119.51 & 119.51 \\
\hline & & & $\mathrm{C} 16-\mathrm{C} 21-\mathrm{C} 22$ & 119.32 & 119.32 \\
\hline & & & C16-C21-H30 & 120.54 & 120.54 \\
\hline & & & C22-C21-H30 & 120.14 & 120.14 \\
\hline & & & $\mathrm{O} 7-\mathrm{C} 22-\mathrm{C} 20$ & 112.76 & 112.76 \\
\hline & & & $\mathrm{O} 7-\mathrm{C} 22-\mathrm{C} 21$ & 126.75 & 126.75 \\
\hline & & & $\mathrm{C} 20-\mathrm{C} 22-\mathrm{C} 21$ & 120.49 & 120.49 \\
\hline Dihedral Angle ( ${ }^{\circ}$ ) & & & Dihedral Angle $\left({ }^{\circ}\right)$ & & \\
\hline $\mathrm{C} 10-\mathrm{O} 1-\mathrm{C} 9-\mathrm{C} 8$ & -0.17 & -0.17 & $\mathrm{C} 13-\mathrm{O} 1-\mathrm{C} 8-\mathrm{C} 9$ & 53.91 & \\
\hline C10-O1-C9-C15 & 179.69 & 179.69 & C13-O1-C8-C10 & 176.26 & \\
\hline C9-O1-C10-C11 & 179.98 & 179.98 & C13-O1-C8-H23 & -65.39 & \\
\hline C9-O1-C10-C12 & 2.19 & 2.19 & $\mathrm{C} 8-\mathrm{O} 1-\mathrm{C} 13-\mathrm{C} 12$ & -22.47 & \\
\hline $\mathrm{H} 28-\mathrm{O} 2-\mathrm{C} 12-\mathrm{C} 10$ & -6.14 & -6.14 & C8-O1-C13-C17 & 159.16 & \\
\hline $\mathrm{H} 28-\mathrm{O} 2-\mathrm{C} 12-\mathrm{C} 13$ & 172.88 & 172.88 & $\mathrm{H} 28-\mathrm{O} 2-\mathrm{C} 9-\mathrm{C} 8$ & 139.37 & \\
\hline H29-O3-C14-C8 & -0.32 & -0.32 & $\mathrm{H} 28-\mathrm{O} 2-\mathrm{C} 9-\mathrm{C} 11$ & 20.74 & \\
\hline $\mathrm{H} 29-\mathrm{O} 3-\mathrm{C} 14-\mathrm{C} 18$ & 179.55 & 179.55 & H28-O2-C9-H24 & -99.50 & \\
\hline $\mathrm{H} 30-\mathrm{O} 5-\mathrm{C} 16-\mathrm{C} 15$ & -179.87 & -179.87 & $\mathrm{H} 31-\mathrm{O} 4-\mathrm{C} 14-\mathrm{C} 12$ & -0.44 & \\
\hline $\mathrm{H} 30-\mathrm{O} 5-\mathrm{C} 16-\mathrm{C} 18$ & 0.16 & 0.16 & $\mathrm{H} 31-\mathrm{O} 4-\mathrm{C} 14-\mathrm{C} 18$ & 178.80 & \\
\hline H31-O6-C20-C17 & -156.92 & -156.92 & H32-O5-C19-C17 & 0.72 & \\
\hline H31-O6-C20-C22 & 25.31 & 25.31 & H32-O5-C19-C18 & 179.78 & \\
\hline
\end{tabular}




\begin{tabular}{|c|c|c|c|c|c|}
\hline & \multicolumn{2}{|c|}{ QCN } & & \multicolumn{2}{|c|}{ TXN } \\
\hline & Lanl2MB & SDD & & Lanl2MB & SDD \\
\hline $\mathrm{H} 32-\mathrm{O} 7-\mathrm{C} 22-\mathrm{C} 20$ & 23.51 & 23.51 & H33-O6-C20-C15 & -179.75 & \\
\hline H32-O7-C22-C21 & -158.35 & -158.35 & H33-O6-C20-C22 & 0.07 & \\
\hline C13-C8-C9-O1 & -0.94 & -0.94 & $\mathrm{H} 34-\mathrm{O} 7-\mathrm{C} 22-\mathrm{C} 20$ & -179.34 & \\
\hline C13-C8-C9-C15 & 179.21 & 179.21 & H34-O7-C22-C21 & 0.78 & \\
\hline $\mathrm{C} 14-\mathrm{C} 8-\mathrm{C} 9-\mathrm{O} 1$ & 179.97 & 179.97 & O1-C8-C9-O2 & -177.59 & \\
\hline C14-C8-C9-C15 & 0.11 & 0.11 & O1-C8-C9-C11 & -57.61 & \\
\hline C9-C8-C13-O4 & -179.45 & -179.45 & O1-C8-C9-H24 & 58.97 & \\
\hline C9-C8-C13-C12 & 0.21 & 0.21 & $\mathrm{C} 10-\mathrm{C} 8-\mathrm{C} 9-\mathrm{O} 2$ & 62.62 & \\
\hline C14-C8-C13-O4 & -0.34 & -0.34 & C10-C8-C9-C11 & -177.40 & \\
\hline C14-C8-C13-C12 & 179.33 & 179.33 & C10-C8-C9-H24 & -60.82 & \\
\hline C9-C8-C14-O3 & 179.68 & 179.68 & $\mathrm{H} 23-\mathrm{C} 8-\mathrm{C} 9-\mathrm{O} 2$ & -57.54 & \\
\hline C9-C8-C14-C18 & -0.20 & -0.20 & $\mathrm{H} 23-\mathrm{C} 8-\mathrm{C} 9-\mathrm{C} 11$ & 62.44 & \\
\hline $\mathrm{C} 13-\mathrm{C} 8-\mathrm{C} 14-\mathrm{O} 3$ & 0.55 & 0.55 & H23-C8-C9-H24 & 179.02 & \\
\hline C13-C8-C14-C18 & -179.33 & -179.33 & O1-C8-C10-C15 & -46.55 & \\
\hline O1-C9-C15-C16 & -179.84 & -179.84 & O1-C8-C10-C16 & 135.25 & \\
\hline $\mathrm{O} 1-\mathrm{C} 9-\mathrm{C} 15-\mathrm{H} 23$ & 0.02 & 0.02 & C9-C8-C10-C15 & 76.13 & \\
\hline C8-C9-C15-C16 & 0.02 & 0.02 & C9-C8-C10-C16 & -102.07 & \\
\hline C8-C9-C15-H23 & 179.88 & 179.88 & $\mathrm{H} 23-\mathrm{C} 8-\mathrm{C} 10-\mathrm{C} 15$ & -164.67 & \\
\hline O1-C10-C11-C17 & -44.48 & -44.48 & $\mathrm{H} 23-\mathrm{C} 8-\mathrm{C} 10-\mathrm{C} 16$ & 17.13 & \\
\hline O1-C10-C11-C19 & 135.78 & 135.78 & O2-C9-C11-O3 & -25.98 & \\
\hline C12-C10-C11-C17 & 133.35 & 133.35 & O2-C9-C11-C12 & 152.94 & \\
\hline C12-C10-C11-C19 & -46.39 & -46.39 & C8-C9-C11-O3 & -147.39 & \\
\hline $\mathrm{O} 1-\mathrm{C} 10-\mathrm{C} 12-\mathrm{O} 2$ & 176.01 & 176.01 & C8-C9-C11-C12 & 31.53 & \\
\hline O1-C10-C12-C13 & -2.97 & -2.97 & $\mathrm{H} 24-\mathrm{C} 9-\mathrm{C} 11-\mathrm{O} 3$ & 96.37 & \\
\hline $\mathrm{C} 11-\mathrm{C} 10-\mathrm{C} 12-\mathrm{O} 2$ & -1.51 & -1.51 & H24-C9-C11-C12 & -84.71 & \\
\hline C11-C10-C12-C13 & 179.51 & 179.51 & $\mathrm{C} 8-\mathrm{C} 10-\mathrm{C} 15-\mathrm{C} 20$ & -178.62 & \\
\hline C10-C11-C17-C20 & -179.28 & -179.28 & $\mathrm{C} 8-\mathrm{C} 10-\mathrm{C} 15-\mathrm{H} 25$ & 2.02 & \\
\hline C10-C11-C17-H24 & -0.32 & -0.32 & C16-C10-C15-C20 & -0.42 & \\
\hline C19-C11-C17-C20 & 0.47 & 0.47 & C16-C10-C15-H25 & -179.78 & \\
\hline C19-C11-C17-H24 & 179.43 & 179.43 & C8-C10-C16-C21 & 178.16 & \\
\hline C10-C11-C19-C21 & -179.84 & -179.84 & $\mathrm{C} 8-\mathrm{C} 10-\mathrm{C} 16-\mathrm{H} 26$ & -1.50 & \\
\hline C10-C11-C19-H26 & -3.09 & -3.09 & C15-C10-C16-C21 & -0.03 & \\
\hline C17-C11-C19-C21 & 0.41 & 0.41 & $\mathrm{C} 15-\mathrm{C} 10-\mathrm{C} 16-\mathrm{H} 26$ & -179.69 & \\
\hline C17-C11-C19-H26 & 177.16 & 177.16 & O3-C11-C12-C13 & 176.36 & \\
\hline $\mathrm{O} 2-\mathrm{C} 12-\mathrm{C} 13-\mathrm{O} 4$ & 2.20 & 2.20 & O3-C11-C12-C14 & -6.43 & \\
\hline $\mathrm{O} 2-\mathrm{C} 12-\mathrm{C} 13-\mathrm{C} 8$ & -177.45 & -177.45 & C9-C11-C12-C13 & -2.51 & \\
\hline $\mathrm{C} 10-\mathrm{C} 12-\mathrm{C} 13-\mathrm{O} 4$ & -178.72 & -178.72 & C9-C11-C12-C14 & 174.70 & \\
\hline C10-C12-C13-C8 & 1.62 & 1.62 & $\mathrm{C} 11-\mathrm{C} 12-\mathrm{C} 13-\mathrm{O} 1$ & -3.93 & \\
\hline $\mathrm{O} 3-\mathrm{C} 14-\mathrm{C} 18-\mathrm{C} 16$ & -179.72 & -179.72 & C11-C12-C13-C17 & 174.42 & \\
\hline $\mathrm{O} 3-\mathrm{C} 14-\mathrm{C} 18-\mathrm{H} 25$ & 0.18 & 0.18 & $\mathrm{C} 14-\mathrm{C} 12-\mathrm{C} 13-\mathrm{O} 1$ & 178.96 & \\
\hline C8-C14-C18-C16 & 0.15 & 0.15 & C14-C12-C13-C17 & -2.70 & \\
\hline C8-C14-C18-H25 & -179.95 & -179.95 & $\mathrm{C} 11-\mathrm{C} 12-\mathrm{C} 14-\mathrm{O} 4$ & 4.19 & \\
\hline C9-C15-C16-O5 & 179.96 & 179.96 & C11-C12-C14-C18 & -175.06 & \\
\hline C9-C15-C16-C18 & -0.07 & -0.07 & $\mathrm{C} 13-\mathrm{C} 12-\mathrm{C} 14-\mathrm{O} 4$ & -178.59 & \\
\hline $\mathrm{H} 23-\mathrm{C} 15-\mathrm{C} 16-\mathrm{O} 5$ & 0.09 & 0.09 & C13-C12-C14-C18 & 2.15 & \\
\hline $\mathrm{H} 23-\mathrm{C} 15-\mathrm{C} 16-\mathrm{C} 18$ & -179.93 & -179.93 & O1-C13-C17-C19 & 179.69 & \\
\hline O5-C16-C18-C14 & 179.95 & 179.95 & O1-C13-C17-H27 & -1.46 & \\
\hline O5-C16-C18-H25 & 0.05 & 0.05 & C12-C13-C17-C19 & 1.26 & \\
\hline $\mathrm{C} 15-\mathrm{C} 16-\mathrm{C} 18-\mathrm{C} 14$ & -0.02 & -0.02 & $\mathrm{C} 12-\mathrm{C} 13-\mathrm{C} 17-\mathrm{H} 27$ & -179.90 & \\
\hline C15-C16-C18-H25 & -179.92 & -179.92 & O4-C14-C18-C19 & -179.43 & \\
\hline C11-C17-C20-O6 & -179.87 & -179.87 & O4-C14-C18-H29 & 0.27 & \\
\hline $\mathrm{C} 11-\mathrm{C} 17-\mathrm{C} 20-\mathrm{C} 22$ & -2.02 & -2.02 & C12-C14-C18-C19 & -0.20 & \\
\hline H24-C17-C20-O6 & 1.16 & 1.16 & C12-C14-C18-H29 & 179.51 & \\
\hline $\mathrm{H} 24-\mathrm{C} 17-\mathrm{C} 20-\mathrm{C} 22$ & 179.00 & 179.00 & C10-C15-C20-O6 & -179.62 & \\
\hline C11-C19-C21-C22 & 0.30 & 0.30 & C10-C15-C20-C22 & 0.57 & \\
\hline C11-C19-C21-H27 & 178.44 & 178.44 & $\mathrm{H} 25-\mathrm{C} 15-\mathrm{C} 20-\mathrm{O} 6$ & -0.25 & \\
\hline H26-C19-C21-C22 & -176.48 & -176.48 & $\mathrm{H} 25-\mathrm{C} 15-\mathrm{C} 20-\mathrm{C} 22$ & 179.94 & \\
\hline H26-C19-C21-H27 & 1.65 & 1.65 & $\mathrm{C} 10-\mathrm{C} 16-\mathrm{C} 21-\mathrm{C} 22$ & 0.33 & \\
\hline O6-C20-C22-O7 & -1.46 & -1.46 & C10-C16-C21-H30 & -179.81 & \\
\hline O6-C20-C22-C21 & -179.57 & -179.57 & $\mathrm{H} 26-\mathrm{C} 16-\mathrm{C} 21-\mathrm{C} 22$ & 179.98 & \\
\hline $\mathrm{C} 17-\mathrm{C} 20-\mathrm{C} 22-\mathrm{O} 7$ & -179.19 & -179.19 & $\mathrm{H} 26-\mathrm{C} 16-\mathrm{C} 21-\mathrm{H} 30$ & -0.16 & \\
\hline C17-C20-C22-C21 & 2.69 & 2.69 & $\mathrm{C} 13-\mathrm{C} 17-\mathrm{C} 19-\mathrm{O} 5$ & 179.71 & \\
\hline $\mathrm{C} 19-\mathrm{C} 21-\mathrm{C} 22-\mathrm{O} 7$ & 179.94 & 179.94 & C13-C17-C19-C18 & 0.70 & \\
\hline C19-C21-C22-C20 & -1.85 & -1.85 & $\mathrm{H} 27-\mathrm{C} 17-\mathrm{C} 19-\mathrm{O} 5$ & 0.87 & \\
\hline $\mathrm{H} 27-\mathrm{C} 21-\mathrm{C} 22-\mathrm{O} 7$ & 1.76 & 1.76 & H27-C17-C19-C18 & -178.14 & \\
\hline
\end{tabular}




\begin{tabular}{|c|c|c|c|c|c|}
\hline & \multicolumn{2}{|c|}{ QCN } & & \multicolumn{2}{|c|}{$\mathbf{T X N}$} \\
\hline & Lanl2MB & SDD & & Lanl2MB & SDD \\
\hline H27-C21-C22-C20 & 179.98 & 179.98 & $\mathrm{C} 14-\mathrm{C} 18-\mathrm{C} 19-\mathrm{O} 5$ & 179.72 & \\
\hline & & & C14-C18-C19-C17 & -1.23 & \\
\hline & & & H29-C18-C19-O5 & 0.01 & \\
\hline & & & H29-C18-C19-C17 & 179.06 & \\
\hline & & & O6-C20-C22-O7 & 0.02 & \\
\hline & & & O6-C20-C22-C21 & 179.91 & \\
\hline & & & $\mathrm{C} 15-\mathrm{C} 20-\mathrm{C} 22-\mathrm{O} 7$ & 179.85 & \\
\hline & & & C15-C20-C22-C21 & -0.27 & \\
\hline & & & $\mathrm{C} 16-\mathrm{C} 21-\mathrm{C} 22-\mathrm{O} 7$ & 179.69 & \\
\hline & & & C16-C21-C22-C20 & -0.17 & \\
\hline & & & H30-C21-C22-O7 & -0.17 & \\
\hline & & & H30-C21-C22-C20 & 179.96 & \\
\hline
\end{tabular}

Table 2. Comparative experimental and theoretical IR peaks indexing and corresponding assignments for both QCN and TXN at WB97XD/SDD.

\begin{tabular}{|c|c|c|c|c|c|c|c|c|c|c|}
\hline & \multirow{4}{*}{ Assignments } \\
\hline \multirow{3}{*}{ No } & \multirow{3}{*}{ Exp. } & \multicolumn{4}{|c|}{ QCN } & \multicolumn{4}{|c|}{ TXN } & \\
\hline & & \multicolumn{2}{|c|}{ Wavenumber $\left(\mathrm{cm}^{-1}\right)$} & \multicolumn{2}{|c|}{ IR_Intensity } & \multicolumn{2}{|c|}{ Wavenumber $\left(\mathrm{cm}^{-1}\right)$} & \multicolumn{2}{|c|}{ IR_Intensity } & \\
\hline & & Unscaled & Scaled & Rel. & Abs. & Unscaled & Scaled & Rel. & Abs. & \\
\hline $\mathbf{1}$ & 3416 & 3329 & 3262 & 275 & 78 & 3455 & 3386 & 178 & 26 & $v O H$ \\
\hline 2 & 2920 & 3256 & 3191 & 4 & 1 & 3102 & 3040 & 23 & 3 & $v_{a s} C H($ Aromatic $)$ \\
\hline 3 & 2851 & 3233 & 3168 & 3 & 1 & 3059 & 2998 & 93 & 14 & $v_{s} C H$ (Aromatic) \\
\hline 4 & 1823 & 1740 & 1705 & 468 & 133 & 1727 & 1692 & 681 & 100 & \multirow[b]{2}{*}{$v c=c$} \\
\hline 5 & 1608 & 1692 & 1658 & 352 & 100 & 1661 & 1628 & 372 & 55 & \\
\hline 6 & 1419 & 1444 & 1415 & 20 & 6 & 1433 & 1404 & 68 & 10 & $\beta O H$ \\
\hline 7 & 1363 & 1406 & 1378 & 179 & 51 & 1415 & 1387 & 90 & 13 & \multirow{2}{*}{$\beta C H$} \\
\hline 8 & 1273 & 1299 & 1273 & 310 & 88 & 1310 & 1284 & 141 & 21 & \\
\hline 9 & 890 & 900 & 882 & 90 & 26 & 907 & 889 & 70 & 10 & $v C-O+v C-C$ \\
\hline 10 & 608 & 606 & 594 & 57 & 16 & 630 & 617 & 9 & 1 & $\gamma \mathrm{OH}+\gamma \mathrm{CH}$ \\
\hline 11 & 434 & 451 & 442 & 53 & 15 & 466 & 457 & 80 & 12 & $\gamma C-O+\gamma C-C$ \\
\hline
\end{tabular}

\subsection{FMOs and NLO analyses.}

The optical behavior and chemical reactivity are mainly determined through bandgap energy and dipole moment for the investigated structures [35-38,42,44,45,54]. Other physical characterization may be computed using $\mathrm{E}_{\mathrm{HOMO}}$ and $\mathrm{E}_{\mathrm{LUMO}}$, such as ionizing-potential IP = $-E_{\text {номо }}$, electron-affinity $E A=-E_{\text {LUMO }}$, chemical-potential $\chi=\left(E_{\text {LUMO }}+E_{\text {Hомо }}\right) / 2$, hardenability $\kappa=\left(E_{\text {LUMO }}-E_{\text {НОмо }}\right) / 2$, electrophilicity $\phi=\chi^{2} / 2 \kappa$ and plasticity $S=1 / \kappa$ [55-57]. To foresee NLO properties for investigated structures, polarizability and 1st-order hyperpolarizability are calculated. The static-polarizability $\left(\alpha_{\text {tot }}\right)$, anisotropic-polarizability $(\Delta \alpha)$ and $1^{\text {st }}$-order hyperpolarizability $\left(\beta_{\text {tot }}\right)$ are determined through the deduced relations $[32,43,58,59]$ :

$$
\begin{aligned}
& \alpha_{\mathrm{tot}}=\frac{\alpha_{\mathrm{xx}}+\alpha_{\mathrm{yy}}+\alpha_{\mathrm{zz}}}{3} \\
& \Delta \alpha=\sqrt{\frac{\left(\alpha_{x x}-\alpha_{y y}\right)^{2}+\left(\alpha_{y y}-\alpha_{z z}\right)^{2}+\left(\alpha_{z z}-\alpha_{x x}\right)^{2}+6 \alpha_{y z}^{2}+6 \alpha_{x y}^{2}+6 \alpha_{x z}^{2}}{2}} \\
& \beta_{t o t}=\sqrt{\left(\beta_{x x x}+\beta_{x y y}+\beta_{x z z}\right)^{2}+\left(\beta_{y y y}+\beta_{y z z}+\beta_{y x x}\right)^{2}+\left(\beta_{z z z}+\beta_{z x x}+\beta_{z y y}\right)^{2}}
\end{aligned}
$$

All computations are completed through WB97XD/SDD level and registered in Table 3. Results showed that both QCN and TXN possess dipole moment (DM=4.79, 3.99 Debye) and bandgap offset $(2.59,2.98 \mathrm{eV})$, respectively. $\mathrm{QCN}$ is more reactive than TXN as a result of its higher dipole-moment and reduced energy gap value (Figure 3). QCN shows the highest hyperpolarizability $\left(\beta_{\text {tot }}=18.44 * 10^{-30} \mathrm{esu}\right)$ than $\mathrm{TXN}$, that is 49 times larger than urea 
$\left[\mathrm{DM}_{\text {urea }}=1.32\right.$ Debye, $\beta_{\text {urea }}=0.3728 * 10^{-30}$ esu $]$ as standard reference $[60,61]$. QCN outstanding NLO response sets a revolutionary recall for NLO manufacture upgrade. Both QCN and TXN are promising candidates as window layers for solar cells and memory switch devices. Previously published like-structures data are compared with QCN and TXN (Table 4). Considering a computational error percent, the accuracy of the obtained results is highly acknowledged.
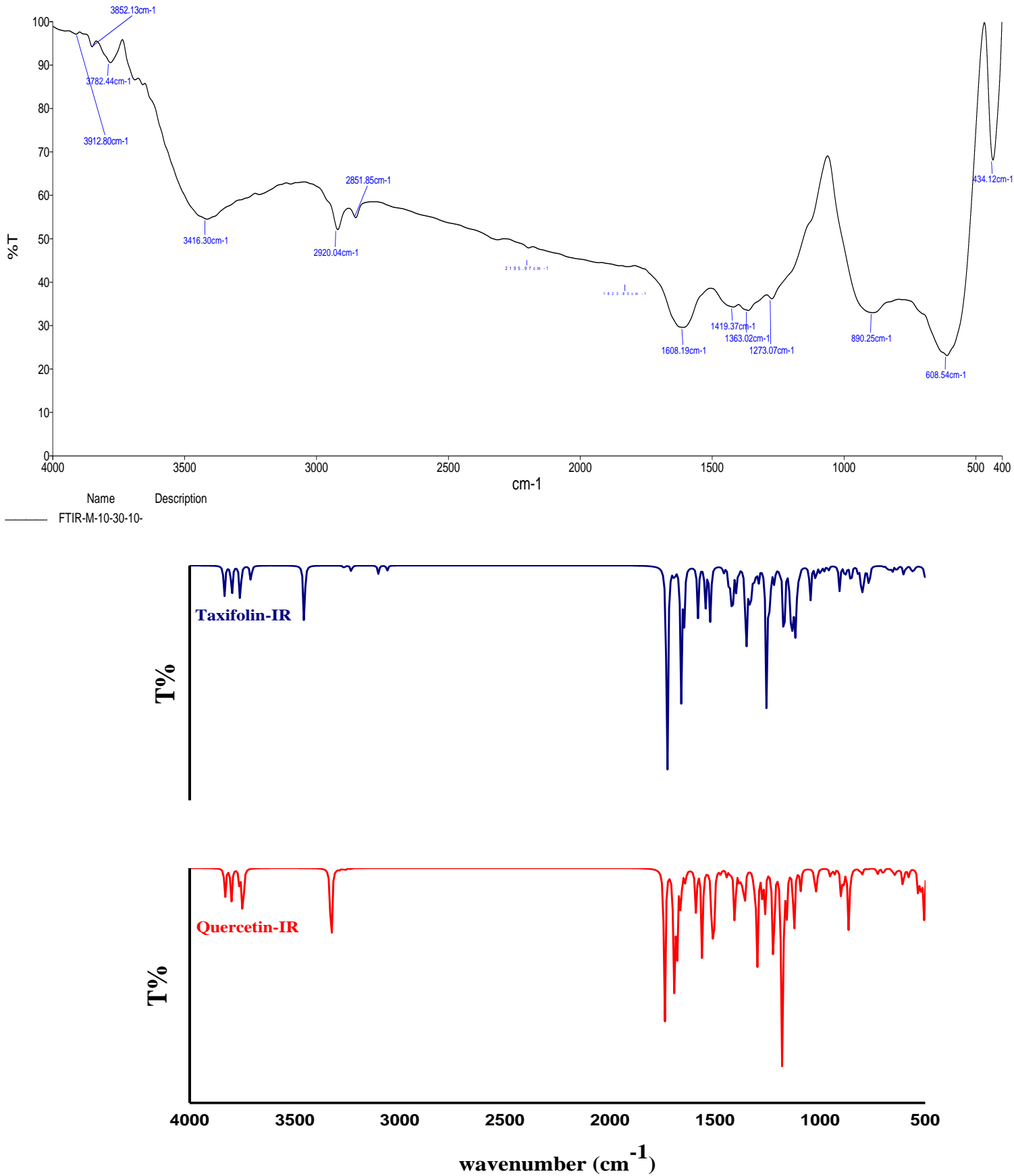

Figure 2. Experimental and Calculated IR spectra for both QCN and TXN at WB97XD/SDD.

Table 3. Static polarizability $\alpha_{\text {tot }}$, polarizability anisotropy $\Delta \alpha$ and $1^{\text {st }}$ hyperpolarizability $\beta_{\text {tot }}$ for QCN and TXN.

\begin{tabular}{c|c|c|c|c|c} 
& \multicolumn{2}{|c|}{$\boldsymbol{\alpha}\left(* \mathbf{1 0}^{-\mathbf{2 4}}\right.$ esu) } & \multicolumn{2}{c}{$\boldsymbol{\beta}\left(* \mathbf{1 0}^{-\mathbf{3 0}}\right.$ esu $)$} \\
\hline & $\mathbf{Q C N}$ & TXN & & $\mathbf{Q C N}$ & TXN \\
\hline $\boldsymbol{\alpha}_{\mathbf{x x}}$ & 42.80 & -4.27 & $\boldsymbol{\beta}_{\mathbf{x x x}}$ & -19.82 & -4.27 \\
\hline $\boldsymbol{\alpha}_{\mathbf{x y}}$ & -0.84 & 2.33 & $\boldsymbol{\beta}_{\mathbf{x x y}}$ & 6.74 & 2.33 \\
\hline $\boldsymbol{\alpha}_{\mathbf{y y}}$ & 29.68 & 3.89 & $\boldsymbol{\beta}_{\mathbf{x y y}}$ & 2.59 & 3.89 \\
\hline $\boldsymbol{\alpha}_{\mathbf{x z}}$ & 0.08 & 3.94 & $\boldsymbol{\beta}_{\mathbf{y y y}}$ & -0.13 & 3.94
\end{tabular}




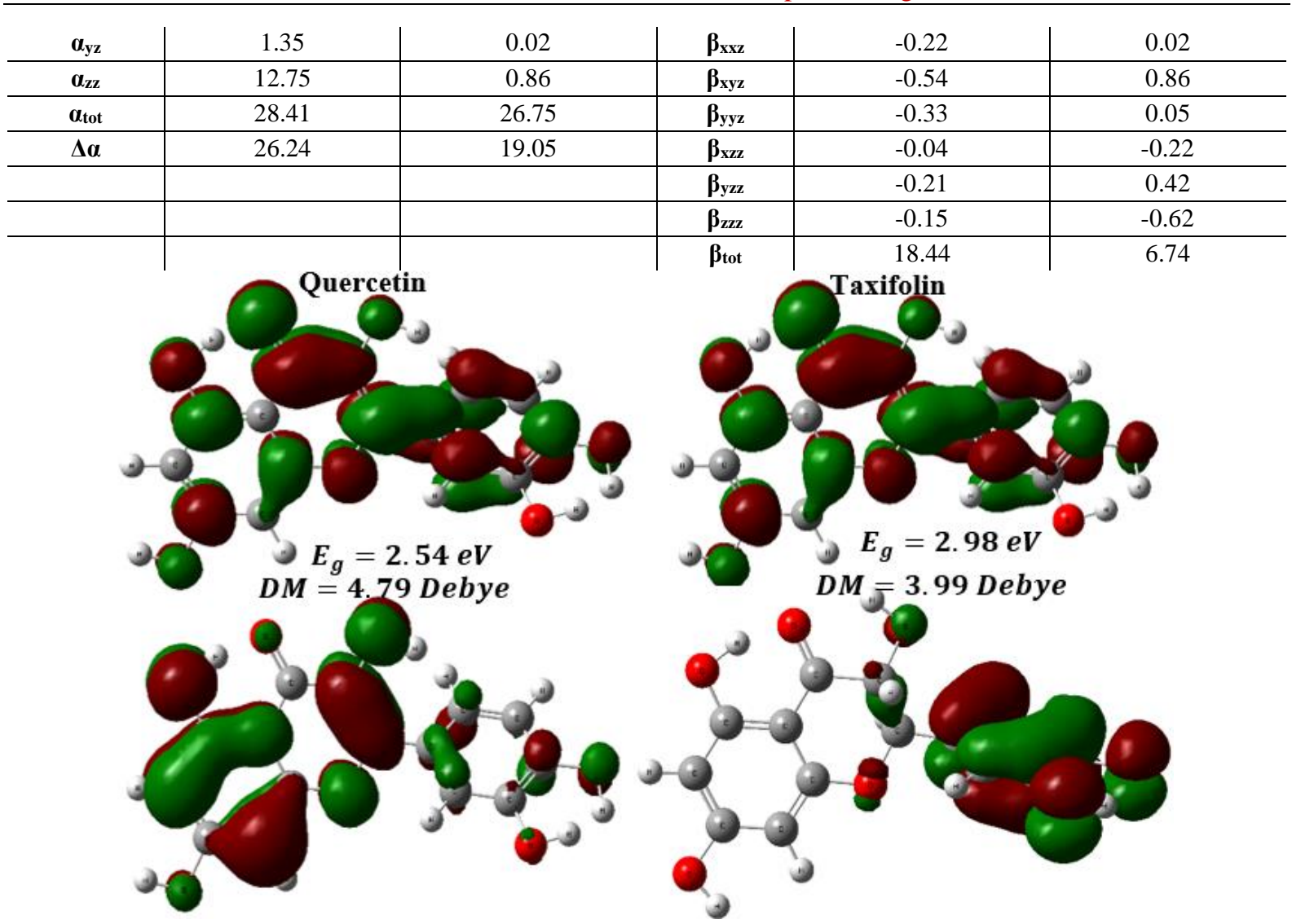

Figure 3. QCN and TXN bandgap offsets at WB97XD/SDD level.

Table 4. A comparative analyses of $\mathrm{E}_{\mathrm{HOMO}}, \mathrm{E}_{\mathrm{LUMO}}$, bandgap offsets, ionizing-potential (IP), electronic-affinity (EA), chemical-potential $(\chi)$, electrophile-index $(\phi)$, hardenability $(\kappa)$, plasticity $(S)$, dipole-moment $(\mathrm{DM})$, Static polarizability $\alpha_{\text {tot }}$, polarizability anisotropy $\Delta \alpha$ and first-order hyperpolarizability $\beta_{\text {tot }}$ for QCN and TXN

\begin{tabular}{|c|c|c|c|c|c|}
\hline & QCN & TXN & Ref.[62] & Ref. [63] & Ref.[59] \\
\hline ELUMO $_{\text {LeV) }}$ & -2.49 & -2.46 & -2.78 & -1.49 & -2.15 \\
\hline Eномо $(\mathrm{eV})$ & -5.03 & -5.44 & -6.33 & -4.89 & -6.14 \\
\hline Energy gap offset $(\mathrm{eV})$ & 2.54 & 2.98 & 3.56 & 3.39 & 3.99 \\
\hline EA $(\mathrm{eV})$ & 2.49 & 2.46 & 2.78 & 1.49 & 2.15 \\
\hline IP $(e V)$ & 5.03 & 5.44 & 6.33 & 4.89 & 6.14 \\
\hline$\kappa(\mathrm{eV})$ & 1.27 & 1.49 & 4.56 & 3.19 & 4.41 \\
\hline$\chi(\mathrm{eV})$ & -3.76 & -3.95 & -4.56 & -3.19 & -4.30 \\
\hline$\phi(\mathrm{eV})$ & 5.56 & 5.24 & 1.78 & 1.69 & 1.99 \\
\hline$S\left(\mathrm{eV}^{-1}\right)$ & 0.79 & 0.67 & 0.28 & 0.29 & 0.50 \\
\hline TDM (Debye) & 4.79 & 3.99 & 5.69 & 6.52 & 2.68 \\
\hline$\alpha_{\text {tot }}\left(* 10^{-24}\right.$ esu $)$ & 28.41 & 26.75 & 34.91 & 48.14 & 19.17 \\
\hline$\Delta \alpha\left(* 10^{-24}\right.$ esu $)$ & 26.24 & 19.05 & 41.85 & 16.04 & 16.80 \\
\hline$\beta_{\text {tot }}\left(* 10^{-30}\right.$ esu $)$ & 18.44 & 6.74 & 2.98 & 29.5 & 7.18 \\
\hline
\end{tabular}

\subsection{NBO analyses.}

NBO is considered an efficient tool in explaining inter and intramolecular bonding and provides a precise style to inspect charge carry or conjugative systems interactions as well [6466]. The $2^{\text {nd }}$ order Fock matrices are performed to investigate the acceptor-donor interplays in NBO for both QCN and TXN. All NBO characterizations for both QCN and TXN are inserted in Table 5. Figure 4 (a) \& (b) predicts the orbital electronegativity for QCN and TXN. Results showed that the highest $E^{(2)}$ values for QCN are $\mathrm{O}_{4}-\mathrm{C}_{13} \rightarrow \mathrm{C}_{8}-\mathrm{C}_{9}$ in $\pi \rightarrow \pi^{*}(288 \mathrm{KJ} / \mathrm{mol}) \&$ $\mathrm{C}_{8}-\mathrm{C}_{9} \rightarrow \mathrm{C}_{14}-\mathrm{C}_{18}$ in $\pi \rightarrow \pi^{*}(325 \mathrm{KJ} / \mathrm{mol})$ whereas for TXN are $\mathrm{C}_{20}-\mathrm{C}_{22} \rightarrow \mathrm{C}_{10}-\mathrm{C}_{15}$ in $\pi$ $\rightarrow \pi^{*}(247 \mathrm{KJ} / \mathrm{mol}) \& \mathrm{C}_{12}-\mathrm{C}_{13} \rightarrow \mathrm{C}_{14}-\mathrm{C}_{18}$ in $\pi \rightarrow \pi^{*}(313 \mathrm{KJ} / \mathrm{mol})$, respectively. 
Table 5. The $2^{\text {nd }}$ order Fock matrix at NBO for both QCN \&TXN.

\begin{tabular}{|c|c|c|c|c|c|c|c|c|c|}
\hline$\frac{\stackrel{\mathscr{J}}{\bar{e}}}{\frac{e}{0}}$ & $\sim$ & 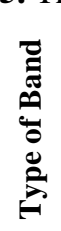 & 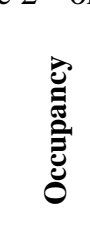 & - & 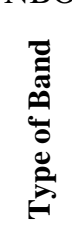 & 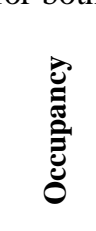 & 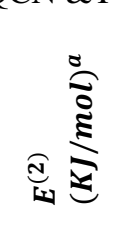 & 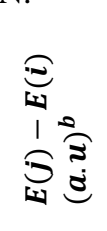 & 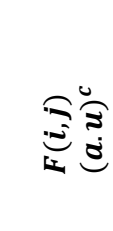 \\
\hline \multirow{19}{*}{ QCN } & \multirow[t]{7}{*}{ C 8 -C 9} & \multirow[t]{7}{*}{$\pi$} & 1.639 & $\mathrm{O} \quad 4-\mathrm{C} 13$ & $\pi^{*}$ & 0.347 & 29.59 & 0.27 & 0.08 \\
\hline & & & 1.639 & C 14 - C 18 & $\pi^{*}$ & 0.369 & 24.45 & 0.29 & 0.075 \\
\hline & & & 1.639 & C 15 - C 16 & $\pi^{*}$ & 0.41 & 12.86 & 0.28 & 0.055 \\
\hline & & & 1.809 & $\mathrm{O} 4-\mathrm{C} 13$ & $\pi^{*}$ & 0.347 & 21.57 & 0.3 & 0.075 \\
\hline & & & 1.666 & C 10 - C 12 & $\pi^{*}$ & 0.266 & 12.19 & 0.28 & 0.053 \\
\hline & & & 1.666 & C 17 - C 20 & $\pi^{*}$ & 0.378 & 20.02 & 0.27 & 0.066 \\
\hline & & & 1.666 & C 21 - C 22 & $\pi^{*}$ & 0.373 & 18.28 & 0.28 & 0.064 \\
\hline & \multirow[t]{2}{*}{ C 14 - C 18} & \multirow[t]{2}{*}{$\pi$} & 1.679 & C 8 - C 9 & $\pi^{*}$ & 0.478 & 12.69 & 0.27 & 0.055 \\
\hline & & & 1.679 & C 15 - C 16 & $\pi^{*}$ & 0.41 & 28.71 & 0.28 & 0.081 \\
\hline & \multirow[t]{2}{*}{ C 15 - C 16} & \multirow[t]{2}{*}{$\pi$} & 1.656 & C 8 - C 9 & $\pi^{*}$ & 0.478 & 29.58 & 0.27 & 0.083 \\
\hline & & & 1.656 & C 14 - C 18 & $\pi^{*}$ & 0.369 & 12.82 & 0.28 & 0.054 \\
\hline & \multirow[t]{2}{*}{ C 17 - C 20} & \multirow[t]{2}{*}{$\pi$} & 1.711 & C 11 - C 19 & $\pi^{*}$ & 0.398 & 17.56 & 0.3 & 0.067 \\
\hline & & & 1.711 & C 21 - C 22 & $\pi^{*}$ & 0.373 & 18.22 & 0.3 & 0.067 \\
\hline & \multirow[t]{2}{*}{ C 21 - C 22} & \multirow[t]{2}{*}{$\pi$} & 1.644 & C 11 - C 19 & $\pi^{*}$ & 0.398 & 21.73 & 0.29 & 0.071 \\
\hline & & & 1.644 & C 17 - C 20 & $\pi^{*}$ & 0.378 & 20.53 & 0.28 & 0.068 \\
\hline & \multirow[t]{2}{*}{$\mathrm{O} 4$ - C 13} & \multirow[t]{2}{*}{$\pi$} & 0.347 & C 8 - C 9 & $\pi^{*}$ & 0.478 & 287.96 & 0.01 & 0.076 \\
\hline & & & 0.347 & C $10-C 12$ & $\pi^{*}$ & 0.266 & 139.67 & 0.02 & 0.076 \\
\hline & C 8 - C 9 & $\pi$ & 0.478 & C 14 - C 18 & $\pi^{*}$ & 0.369 & 325.39 & 0.01 & 0.079 \\
\hline & C 17 - C 20 & $\pi$ & 0.378 & C 11 - C 19 & $\pi^{*}$ & 0.398 & 262.17 & 0.01 & 0.078 \\
\hline \multirow{12}{*}{ TXN } & \multirow{2}{*}{ C $10-$ C 15} & \multirow[t]{2}{*}{$\pi$} & 1.683 & C $16-C 21$ & $\pi^{*}$ & 0.358 & 20.21 & 0.28 & 0.068 \\
\hline & & & 1.683 & C 20 - C 22 & $\pi^{*}$ & 0.412 & 20.71 & 0.27 & 0.069 \\
\hline & \multirow[t]{2}{*}{ C 12 - C 13} & \multirow[t]{2}{*}{$\pi$} & 1.619 & $\mathrm{O} 3-\mathrm{C} 11$ & $\pi^{*}$ & 0.229 & 33.9 & 0.27 & 0.089 \\
\hline & & & 1.619 & C 14 - C 18 & $\pi^{*}$ & 0.345 & 25.15 & 0.29 & 0.077 \\
\hline & C 14 - C 18 & $\pi$ & 1.65 & C 17 - C 19 & $\pi^{*}$ & 0.403 & 27.64 & 0.27 & 0.079 \\
\hline & \multirow[t]{2}{*}{ C 16 - C 21} & \multirow[t]{2}{*}{$\pi$} & 1.691 & C $10-C 15$ & $\pi^{*}$ & 0.372 & 19.98 & 0.29 & 0.069 \\
\hline & & & 1.691 & C 20 - C 22 & $\pi^{*}$ & 0.412 & 20.59 & 0.27 & 0.069 \\
\hline & C 17 - C 19 & $\pi$ & 1.64 & C 12 - C 13 & $\pi^{*}$ & 0.476 & 26.65 & 0.28 & 0.079 \\
\hline & \multirow[t]{2}{*}{ C 20 - C 22} & \multirow[t]{2}{*}{$\pi$} & 1.636 & C 10 - C 15 & $\pi^{*}$ & 0.372 & 19.15 & 0.3 & 0.068 \\
\hline & & & 1.636 & C 16 - C 21 & $\pi^{*}$ & 0.358 & 19.92 & 0.29 & 0.068 \\
\hline & C 12 - C 13 & $\pi$ & 0.476 & C $14-\mathrm{C} 18$ & $\pi^{*}$ & 0.345 & 313.1 & 0.01 & 0.081 \\
\hline & C 20 - C 22 & $\pi$ & 0.412 & C $10-C 15$ & $\pi^{*}$ & 0.372 & 247.3 & 0.01 & 0.082 \\
\hline
\end{tabular}

Donor: $i$; Acceptor: $j$

${ }^{\mathrm{a}} E^{(2)}$ means hyper-conjugative energy interplays.

${ }^{\mathrm{b}}$ Differential energy among $\operatorname{donor}(i)$ and acceptor $(j)$ Orbitals at NBO

${ }^{\mathrm{c}} F(i, i)$ is the Fock matrix

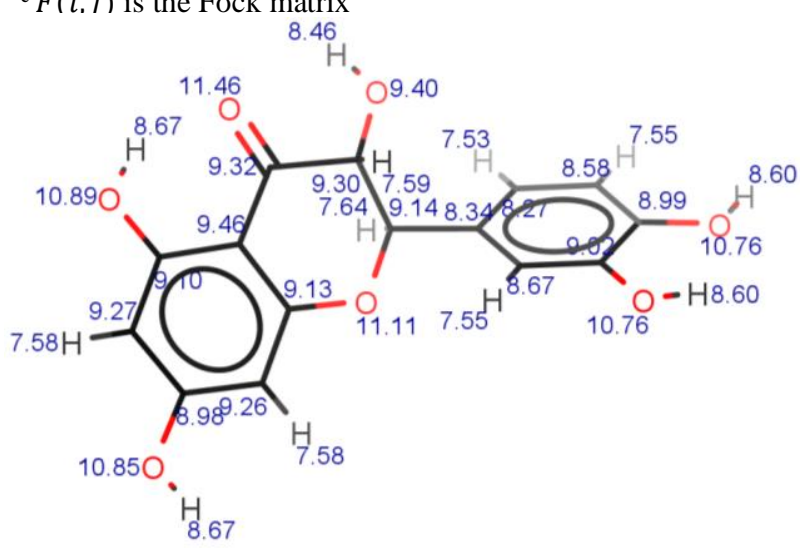

(a) $\mathrm{QCN}$

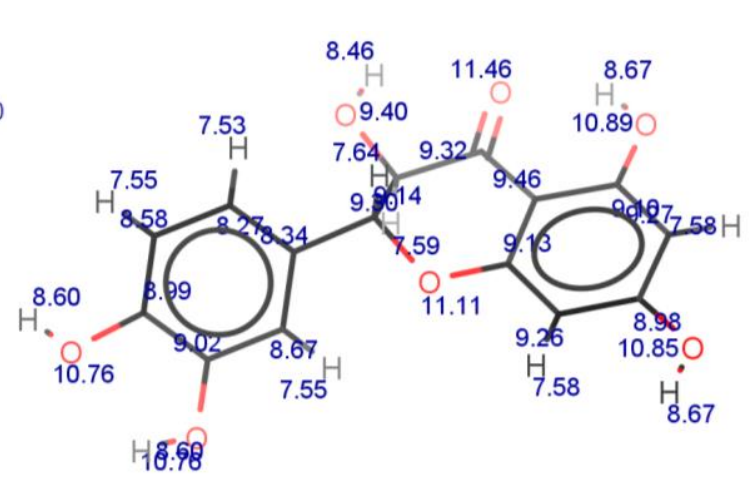

(b) TXN

Figure 4. Orbital electronegativity for (a) QCN and (b) TXN.

\subsection{UV-Vis Absorption calculations.}

The optical properties of QCN and TXN are carried out using TD-DFT/B3LYP/6$311 \mathrm{G}(\mathrm{d}, \mathrm{p})++$, which are applied on optimal QCN and TXN structures. The UV-Vis spectra for QCN and TXN are shown in Figures 5 (a)\&(b). The theoretical UV-Vis absorption spectra are 
reported within the range of $200-800 \mathrm{~nm}$. The dominant absorption peak in QCN is situated at $317 \mathrm{~nm}$, whereas TXN is allocated at $270 \mathrm{~nm}$ (blue shift due to two $\mathrm{H}$ antennae attached to aromatic moiety). The compositions of distinguished excitations, oscillator strength, and excitation energy are shown in Table 6. Figure 6 shows the observed UV-Vis spectrum for Mangifera Indica leaves extract. Four major absorption peaks are detected at 210, 269, 317, and $360 \mathrm{~nm}$ for Mangifera Indica leaves extract. The prominent transition in QCN is mainly due to $H_{-1} \rightarrow L$ (transition coefficient $\sim 0.85$ ) while for TXN is produced by $H_{-3} \rightarrow L$ (transition coefficient 0.88 ). Eventually, QCN seemed to be optically active than TXN due to $\pi$ - electron pair resonating along aromatic moiety and pushing the Fermi level up close to the conduction band, which made electron excitation much easier than in TXN.

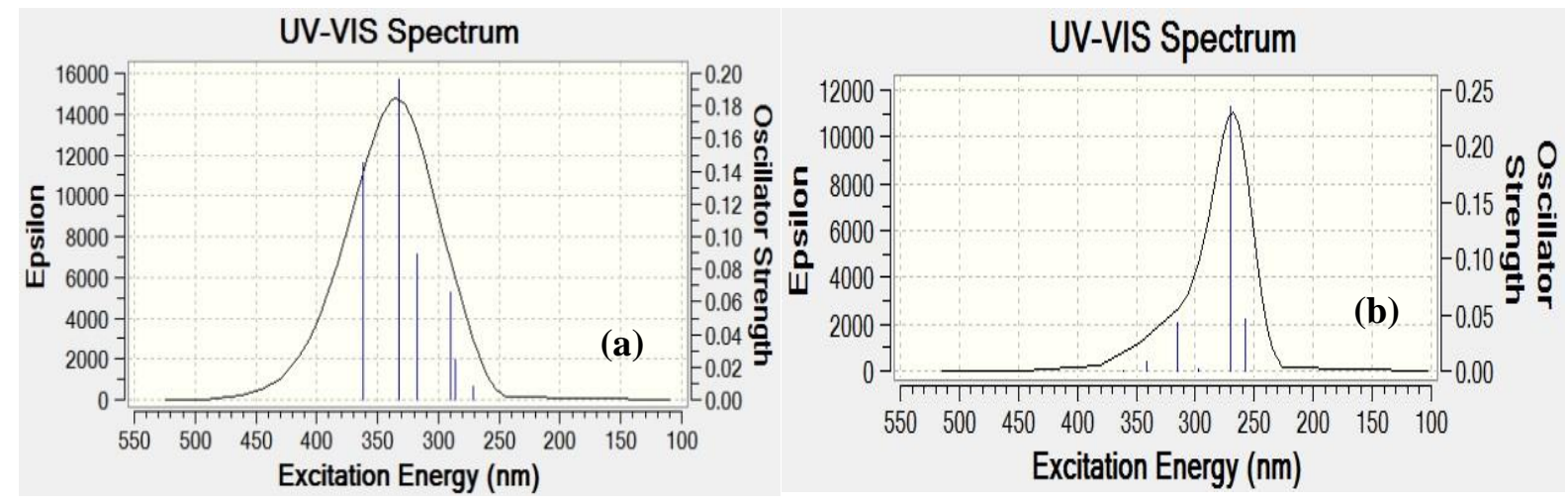

Figure 5. Theoretical UV-Vis spectra for (a) QCN) \& (b) TXN.

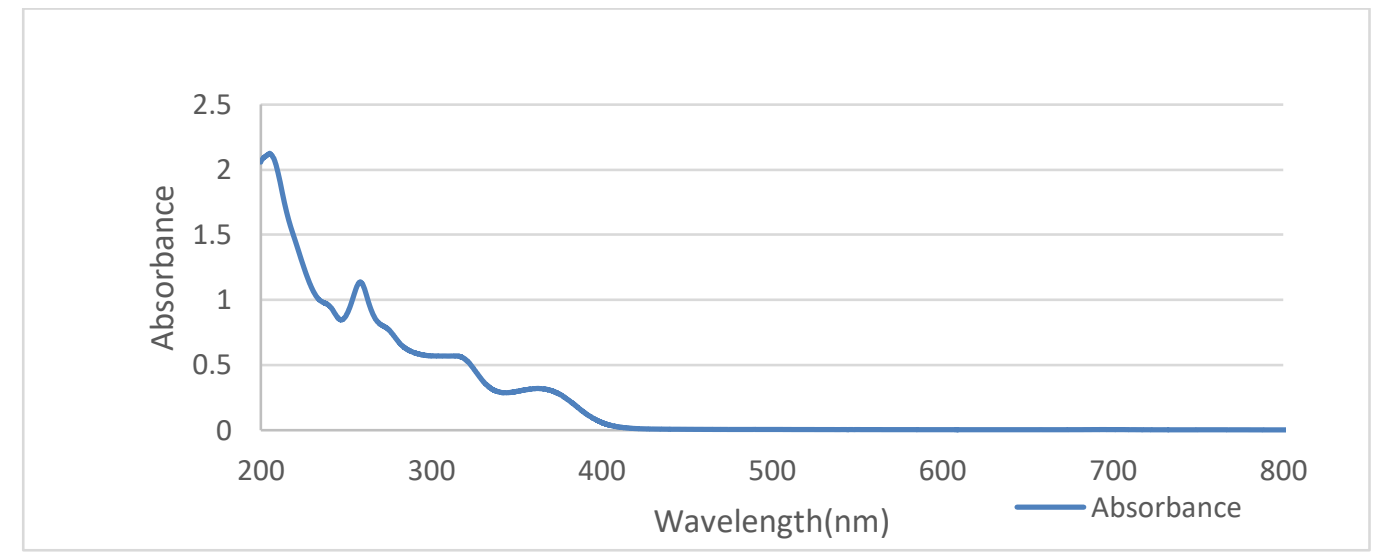

Figure 6. Observed UV-Vis Spectrum for Mangifera Indica leaves extract.

Table 6. Oscillator strength (f), wavelength $(\lambda)$, excitation energy $\left(E_{x}\right)$, and transition compositions of prominent transitions in QCN and TXN.

\begin{tabular}{|c|c|c|c|c|}
\hline & $\lambda(\mathbf{n m})$ & $E_{x}(e V)$ & $f(\mathbf{a . u})$ & Transition composition \\
\hline \multirow{7}{*}{ QCN } & 362 & 3.42 & 0.15 & $\begin{aligned} \mathrm{H} & \rightarrow \mathrm{L}(57 \%) \\
\mathrm{H}_{-3} & \rightarrow \mathrm{L}(25 \%), \\
\mathrm{H}_{-1} & \rightarrow \mathrm{L}(10 \%)\end{aligned}$ \\
\hline & 332 & 3.74 & 0.2 & $\begin{aligned} \mathrm{H}_{-3} & \rightarrow \mathrm{L}(58 \%), \\
\mathrm{H} & \rightarrow \mathrm{L}(34 \%)\end{aligned}$ \\
\hline & 317 & 3.91 & 0.09 & $\mathrm{H}_{-1} \rightarrow \mathrm{L}(85 \%)$ \\
\hline & 290 & 4.28 & 0.07 & $\begin{aligned} \mathrm{H}_{-4} & \rightarrow \mathrm{L}(16 \%), \\
\mathrm{H}_{-2} & \rightarrow \mathrm{L}(67 \%)\end{aligned}$ \\
\hline & 286 & 4.33 & 0.02 & $\begin{array}{r}\mathrm{H}_{-4} \rightarrow \mathrm{L}(41 \%), \\
\mathrm{H}_{-2} \rightarrow \mathrm{L}(13 \%) \\
\mathrm{H} \rightarrow \mathrm{L}_{+1}(29 \%)\end{array}$ \\
\hline & 272 & 4.56 & 0.01 & $\begin{array}{c}\mathrm{H}_{-4} \rightarrow \mathrm{L}(21 \%) \\
\mathrm{H}_{-2} \rightarrow \mathrm{L}_{+1}(52 \%)\end{array}$ \\
\hline & 361 & 3.44 & 0 & $\begin{aligned} \mathrm{H} & \rightarrow \mathrm{L}(46 \%) \\
\mathrm{H}_{-4} & \rightarrow \mathrm{L}(26 \%) \\
\mathrm{H}_{-1} & \rightarrow \mathrm{L}(25 \%)\end{aligned}$ \\
\hline
\end{tabular}




\begin{tabular}{|c|c|c|c|c|}
\hline & $\lambda(\mathrm{nm})$ & $E_{x}(e V)$ & $f(\mathbf{a} . \mathbf{u})$ & Transition composition \\
\hline \multirow[t]{5}{*}{ TXN } & 341 & 3.63 & 0.01 & $\begin{array}{c}\mathrm{H} \rightarrow \mathrm{L}(53 \%) \\
\mathrm{H}_{-4} \rightarrow \mathrm{L}(20 \%) \\
\mathrm{H}_{-1} \rightarrow \mathrm{L}(25 \%)\end{array}$ \\
\hline & 315 & 3.93 & 0.04 & $\begin{aligned} \mathrm{H}_{-4} & \rightarrow \mathrm{L}(26 \%), \\
\mathrm{H}_{-2} & \rightarrow \mathrm{L}(36 \%) \\
\mathrm{H}_{-1} & \rightarrow \mathrm{L}(33 \%),\end{aligned}$ \\
\hline & 298 & 4.17 & 0 & $\begin{aligned} \mathrm{H}_{-4} & \rightarrow \mathrm{L}(23 \%), \\
\mathrm{H}_{-2} & \rightarrow \mathrm{L}(62 \%) \\
\mathrm{H}_{-1} & \rightarrow \mathrm{L}(14 \%),\end{aligned}$ \\
\hline & 270 & 4.59 & 0.23 & $\mathrm{H}_{-3} \rightarrow \mathrm{L}(88 \%)$ \\
\hline & 257 & 4.82 & 0.05 & $\begin{array}{c}\mathrm{H}_{-2} \rightarrow \mathrm{L}_{+5}(10 \%), \\
\mathrm{H} \rightarrow \mathrm{L}_{+1}(18 \%) \\
\mathrm{H} \rightarrow \mathrm{L}_{+2}(65 \%)\end{array}$ \\
\hline
\end{tabular}

L; LUMO, H; HOMO (Highest occupied molecular orbitals).

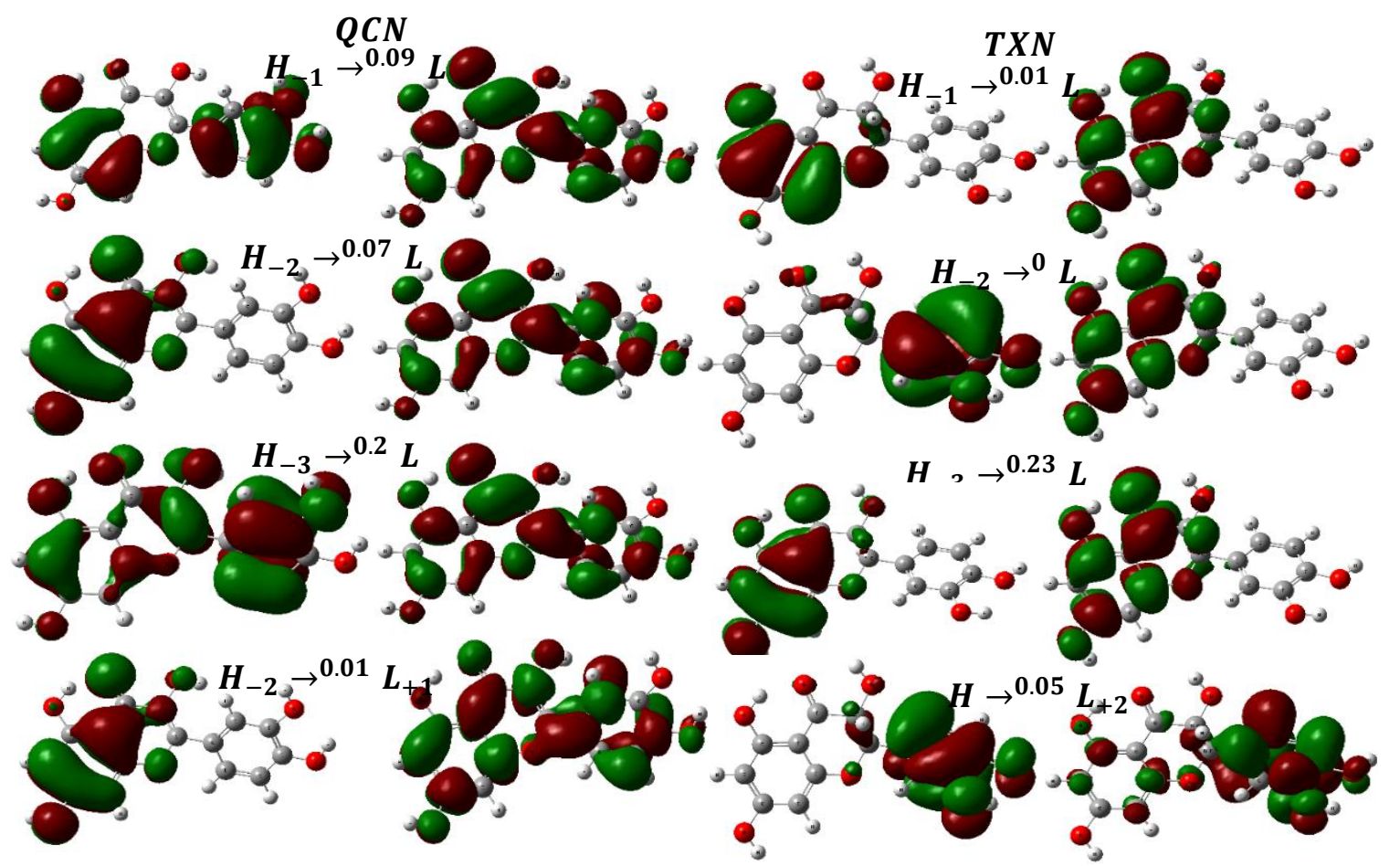

Figure 7. Simulated dominant transitions for both QCN and TXN.

The dominant transitions compositions for both QCN and TXN are shown in Figure 7. It can be noticed that charge carry is higher in QCN than TXN, which in turn stimulates high absorption efficiency for QCN.

\section{Conclusions}

The authors investigate a novel synthesis process for QCN, and TXN extracts from Mangifera indica using Ag nanoparticles. Both FT-IR and UV-Vis spectra are performed for refined Mangifera extracts with high precision. Either IR or UV-Vis spectra are simulated using G09 software utilizing DFT. Results show an excellent agreement with measured data, especially with QCN. Also, QCN shows the highest hyperpolarizability ( $\beta$ tot $=18.44 * 10-30$ esu) than TXN. QCN outstanding NLO response sets a revolutionary recall for the NLO manufacture upgrade. In addition, QCN seemed to be more optically active than TXN due to $\pi$ - electron pair resonating along with aromatic moiety and pushing the fermi level up close to the conduction band, which made the electron excitation much larger and easier than in TXN. 
Eventually, Both QCN and TXN are promising candidates as window layers for solar cells and memory switch devices.

\section{Funding}

This research received no external funding.

\section{Acknowledgments}

This research has no acknowledgment.

\section{Conflicts of Interest}

The authors declare no conflict of interest.

\section{References}

1. Lara, H.H.; Ayala-Núñez, N.V.; Turrent, L.d.C.I.; Padilla, C.R. Bactericidal effect of silver nanoparticles against multidrug-resistant bacteria. World Journal of Microbiology Biotechnology 2010, 26, 615-621, https://doi.org/10.1007/s11274-009-0211-3.

2. Ahmadi, S. The importance of silver nanoparticles in human life. Advances in Applied NanoBio-Technologies 2020, 1, 5-9, https://www.dormaj.org/index.php/AANBT/article/view/20.

3. Ghiuță, I.; Cristea, D. Silver nanoparticles for delivery purposes. Nanoengineered Biomaterials for Advanced Drug Delivery 2020, 347-371, https://doi.org/10.1016/B978-0-08-102985-5.00015-2.

4. Lv, X.; Tang, Y.; Tian, Q.; Wang, Y.; Ding, T. Ultra-stretchable membrane with high electrical and thermal conductivity via electrospinning and in-situ nanosilver deposition. Composites Science and Technology 2020, 200, 108414, https://doi.org/10.1016/j.compscitech.2020.108414.

5. Wu, Y.; Zhang, X.; Negi, A.; He, J.; Hu, G.; Tian, S.; Liu, J. Synergistic Effects of Boron Nitride (BN) Nanosheets and Silver (Ag) Nanoparticles on Thermal Conductivity and Electrical Properties of Epoxy Nanocomposites. Polymers 2020, 12, 426, https://doi.org/10.3390/polym12020426.

6. El-Nahrawy, A.M.; Abou Hammad, A.B.; Khattab, T.A.; Haroun, A.; Kamel, S. Development of electrically conductive nanocomposites from cellulose nanowhiskers, polypyrrole and silver nanoparticles assisted with Nickel (III) oxide nanoparticles. Reactive and Functional Polymers 2020, 149, 104533, https://doi.org/10.1016/j.reactfunctpolym.2020.104533.

7. Lalegani, Z.; Ebrahimi, S.S.; Hamawandi, B.; La Spada, L.; Toprak, M. Modeling, design, and synthesis of gram-scale monodispersed silver nanoparticles using microwave-assisted polyol process for metamaterial applications. Optical Materials 2020, 108, 110381, https://doi.org/10.1016/j.optmat.2020.110381.

8. Gholami, T.; Salavati-Niasari, M.; Varshoy, S. Investigation of the electrochemical hydrogen storage and photocatalytic properties of $\mathrm{CoAl} 2 \mathrm{O} 4$ pigment: green synthesis and characterization. International Journal of Hydrogen Energy 2016, 41, 9418-9426, https://doi.org/10.1016/j.ijhydene.2016.03.144.

9. Razavi, F.S.; Hajizadeh-Oghaz, M.; Amiri, O.; Morassaei, M.S.; Salavati-Niasari, M. Barium cobaltite nanoparticles: Sol-gel synthesis and characterization and their electrochemical hydrogen storage properties. International Journal of Hydrogen Energy 2021, 46, 886-895, https://doi.org/10.1016/j.ijhydene.2020.09.196.

10. Mamun, M.S.A.; Hossain, M.; Naime, J.; Dhar, P. Plasmonic Nanosilver Synthesis Using Sonneratia apetala Fruit Extract and Their Catalytic Activity in Organic Dye Degradation. Journal of Chemical Health Risks 2020, 10, 287-296, https://doi.org/10.22034/jchr.2020.1904366.1155.

11. Ghosh, K.; Tyagi, S.; Gupta, A. Analytical and Immunopharmacological Studies of Flavonoids: Mangifera Indica and Syzygium Cumini. Biomedical and Pharmacology Journal 2020, 13, 899-906, https://dx.doi.org/10.13005/bpj/1957.

12. Mirza, B.; Croley, C.R.; Ahmad, M.; Pumarol, J.; Das, N.; Sethi, G.; Bishayee, A. Mango (Mangifera indica L.): a magnificent plant with cancer preventive and anticancer therapeutic potential. Critical Reviews in Food Science and Nutrition 2021, 61, 2125-2151, https://doi.org/10.1080/10408398.2020.1771678.

13. Tchoumi, L.T.; Nchouwet, M.; Kamani, S.P.; Nana, W.Y.; Djimeli, R.D.; Kamanyi, A.; Ngnokam, S. Antimicrobial and antidiarrhoeal activities of aqueous and methanolic extracts of Mangifera indica Linn stem 
bark (Anarcadiaceae) in Wistar rats. Advances in Traditional Medicine 2021, 21, 485-498, https://doi.org/10.1007/s13596-020-00470-6.

14. Lakhanpal, P.; Rai, D. Quercetin: a versatile flavonoid. Internet Journal of Medical Update 2007, 2, 22-37, https://www.akspublication.com/Paper05_Jul-Dec2007_.pdf.

15. Burdeos, G.C.; Blank, R.; Wolffram, S. Influence of quercetin on the global DNA methylation pattern in pigs. Food Function 2020, 11, 7421-7426, https://doi.org/10.1039/D0FO00896F.

16. Khan, H.; Ullah, H.; Aschner, M.; Cheang, W.S.; Akkol, E. Neuroprotective effects of quercetin in Alzheimer's disease. Biomolecules 2020, 10, 59, https://doi.org/10.3390/biom10010059.

17. Kang, J.-T.; Moon, J.H.; Choi, J.-Y.; Park, S.J.; Kim, S.J.; Saadeldin, I.M.; Lee, B. Effect of antioxidant flavonoids (quercetin and taxifolin) on in vitro maturation of porcine oocytes. Asian-Australas Journal Animal Sciences 2016, 29, 352-358, https://doi.org/10.5713/ajas.15.0341.

18. Salama, S.A.; Kabel, A. Taxifolin ameliorates iron overload-induced hepatocellular injury: Modulating PI3K/AKT and p38 MAPK signaling, inflammatory response, and hepatocellular regeneration. ChemicoBiological Interactions 2020, 330, 109230, https://doi.org/10.1016/j.cbi.2020.109230.

19. Sunil, C.; Xu, B. An insight into the health-promoting effects of taxifolin (dihydroquercetin). Phytochemistry 2019, 166, 112066, https://doi.org/10.1016/j.phytochem.2019.112066.

20. Cui, Y.; Zhang, X.; Yin, K.; Qi, X.; Zhang, Y.; Zhang, J.; Li, S.; Lin, H. Dibutyl phthalate-induced oxidative stress, inflammation and apoptosis in grass carp hepatocytes and the therapeutic use of taxifolin. Science of The Total Environment 2020, 142880, https://doi.org/10.1016/j.scitotenv.2020.142880.

21. Vrba, J.; Papoušková, B.; Kosina, P.; Lněničková, K.; Valentová, K.; Ulrichová, J. Identification of Human Sulfotransferases Active towards Silymarin Flavonolignans and Taxifolin. Metabolites 2020, 10, 329, https://doi.org/10.3390/metabo10080329.

22. Šimunková, M.; Valko, M.; Bučinský, L.; Malček, M. Structure functionality relationship of flavonoids (myricetin, morin, taxifolin and 3', 4'-dihydroxyflavone). A computational study via the cupric ion probe. Journal of Molecular Structure 2020, 1222, 128923, https://doi.org/10.1016/j.molstruc.2020.128923.

23. Wang, R.; Zhu, X.; Wang, Q.; Li, X.; Wang, E.; Zhao, Q.; Wang, Q.; Cao, H. The anti-tumor effect of taxifolin on lung cancer via suppressing stemness and epithelial-mesenchymal transition in vitro and oncogenesis in nude mice. Annals Translational Medicine 2020, 8, 590, https://doi.org/10.21037/atm-20-3329.

24. Zhou, W.; Liu, Z.; Wang, M.; Chen, D.; Zhou, L.; Guo, L. Taxifolin inhibits the development of scar cell carcinoma by inducing apoptosis, cell cycle arrest, and suppression of PI3K/AKT/mTOR pathway. Journal of BUON 2019, 24, 853-858, https://www.jbuon.com/archive/24-2-853.pdf.

25. Yeh, W.-J.; Hsia, S.-M.; Lee, W.-H.; Wu, C.-H. Polyphenols with antiglycation activity and mechanisms of action: A review of recent findings. Journal of Food and Drug Analysis 2017, 25, 84-92, https://doi.org/10.1016/j.jfda.2016.10.017.

26. García-Conesa, M.-T.; Larrosa, M. Polyphenol-Rich Foods for Human Health and Disease. Nutrients 2020, 12, 400, https://doi.org/10.3390/nu12020400.

27. M. J. Frisch, G.W.T., H. B. Schlegel, G. E. Scuseria, M. A. Robb, J. R. Cheeseman, J. A. Montgomery, Jr., T. Vreven, K. N. Kudin, J.C. Burant, J. M. Millam, S.S. Iyengar, J. Tomasi, V. Barone, B. Mennucci, M. Cossi, G. Scalmani, N. Rega, G. A. Petersson, H. Nakatsuji, M. Hada, M.Ehara, K. Toyota, R. Fukuda, J. Hasegawa, M. Ishida, T. Nakajima, Y. Honda, O. Kitao, H. Nakai, M. Klene, X. Li, J. E. Knox, H.P. Hratchian, J.B. Cross, C. Adamo, J. Jaramillo, R. Gomperts, R. E. Stratmann, O. Yazyev, A.J. Austin, R.Cammi, C. Pomelli, J.W. Ochterski, P.Y. Ayala, K. Morokuma, G.A. Voth, P. Salvador, J. J. Dannenberg, V.G. Zakrzewski, S. Dapprich, A.D. Daniels, M.C. Strain, O. Farkas, D.K. Malick, A.D. Rabuck, K. Raghavachari, J. B. Foresman, J. V. Ortiz, Q. Cui, A.G. Baboul, S. Clifford, J. Cioslowski, B. B. Stefanov, G. Liu, A. Liashenko, P. Piskorz, I. Komaromi, R.L. Martin, D.J.Fox, T. Keith, M. A. Al-Laham, C.Y. Peng, A. Nanayakkara, M. Challacombe, P. M. W. Gill, B. Johnson, W. Chen, M. W. Wong, C. Gonzalez, J. A. Pople. Gaussian09, Revision A. Inc. Wallingford CT 2009, https://gaussian.com/g09citation/.

28. Frisch, A.; Dennington, R.; Keith, T.; Millam, J.; Nielsen, A.; Holder, A.; Hiscocks, J. Gauss view version 5 user manual. Gaussian Inc., Wallingford, CT, USA 2009,

29. El-Mansy, M.A.; Osman, O.; Mahmoud, A.A.; Elhaes, H.; Ibrahim, M.A. Computational notes on the molecular modeling analyses of flutamide. Letters in Applied NanoBioScience 2020, 9, 1099-1102, https://doi.org/10.33263/LIANBS93.11471155.

30. El-Mansy, M.A.; Bayoumy, A.M.; Ezzat, H.; El-Sayed, N.; Elhaes, H.; Osman, O.; Mahmoud, A.A.; Ibrahim, A. Modeling the Effect of Hydration on the Electronic and Vibrational Properties of AZT. Biointerface Research in Applied Chemistry 2021, 11, 9253-9265, https://doi.org/10.33263/BRIAC112.92539265. 
31. El-Mansy, M.A. Quantum chemical studies on structural, vibrational, nonlinear optical properties and chemical reactivity of indigo carmine dye. Spectrochimica Acta Part A: Molecular and Biomolecular Spectroscopy 2017, 183, 284-290, https://doi.org/10.1016/j.saa.2017.04.047.

32. Soliman, H.; Ibrahim, M.; El-Mansy, M.A.; Atef, S. Structural and optical study of nanostructure of 4cyanopyranoquinolinedione (CPQ) thin films. Optical Materials 2017, 72, 122-129, https://doi.org/10.1016/j.optmat.2017.05.052.

33. El-Mansy, M.A.; El-Bana, M.; Fouad, S. On the spectroscopic analyses of 3-Hydroxy-1-Phenyl-Pyridazin-6 (2H) one (HPHP): A comparative experimental and computational study. Spectrochimica Acta Part A: Molecular and Biomolecular Spectroscopy 2017, 176, 99-105, https://doi.org/10.1016/j.saa.2016.12.040.

34. Gulácsi, M.; El-Mansy, M.A.; Gulácsi, Z. Electron-phonon interactions in conducting polymers. Philosophical Magazine Letters 2016, 96, 67-75, https://doi.org/10.1080/09500839.2016.1150611.

35. Ibrahim, M.; El-Barbary, A.A.; El-Nahass, M.; Kamel, M.; El-Mansy, M.A.; Asiri, A.M. On the spectroscopic analyses of (E)-3-(dicyclopropyl methylene)-dihydro-4-[1-(2, 5 dimethylfuran-3-yl) ethylidene] furan-2, 5dione. Spectrochimica Acta Part A: Molecular Biomolecular Spectroscopy 2012, 87, 202-208, https://doi.org/10.1016/j.saa.2011.11.039.

36. El-Nahass, M.; Kamel, M.; El-Barbary, A.; El-Mansy, M.A.; Ibrahim, M. FT-IR spectroscopic analyses of 3Methyl-5-Pyrazolone (MP). Spectrochimica Acta Part A: Molecular Biomolecular Spectroscopy 2013, 111, 37-41, https://doi.org/10.1016/j.saa.2013.03.072.

37. El-Mansy, M.A.; El-Nahass, M.; Khusayfan, N.; El-Menyawy, E. DFT approach for FT-IR spectra and HOMO-LUMO energy gap for N-(p-dimethylaminobenzylidene)-p-nitroaniline (DBN). Spectrochimica Acta Part A: Molecular Biomolecular Spectroscopy 2013, 111, 217-222, https://doi.org/10.1016/j.saa.2013.04.018.

38. Ibrahim, M.; El-Nahass, M.; Kamel, M.; El-Barbary, A.; Wagner, B.; El-Mansy, M.A. On the spectroscopic analyses of thioindigo dye. Spectrochimica Acta Part A: Molecular Biomolecular Spectroscopy 2013, 113, 332-336, https://doi.org/10.1016/j.saa.2013.05.014.

39. Soliman, H.; Eid, K.M.; Ali, H.; El-Mansy, M.A.; Atef, S. FT-IR spectroscopic analyses of 2-(2furanylmethylene) propanedinitrile. Spectrochimica Acta Part A: Molecular Biomolecular Spectroscopy 2013, 105, 545-549, https://doi.org/10.1016/j.saa.2012.12.051.

40. Soliman, H.; Eid, K.M.; Ali, H.; Atef, S.; El-Mansy, M.A. Vibrational spectroscopic analysis of 2-chloro-5(2, 5-dimethoxy-benzylidene)-1, 3-diethyl-dihydro-pyrimidine-4, 6 (1H, 5H)-dione. Spectrochimica Acta Part A: Molecular Biomolecular Spectroscopy 2012, 97, 1079-1084, https://doi.org/10.1016/j.saa.2012.07.104.

41. Ismail, M.; Morsy, G.; Mohamed, H.; El-Mansy, M.A.; Abd-Alrazk, M. FT-IR spectroscopic analyses of 4hydroxy-1-methyl-3-[2-nitro-2-oxoacetyl-2 (1H) quinolinone (HMNOQ). Spectrochimica Acta Part A: Molecular Biomolecular Spectroscopy 2013, 113, 191-195, https://doi.org/10.1016/j.saa.2013.04.117.

42. El-Barbary, A.; El-Nahass, M.; Kamel, M.; El-Mansy, M.A. FT-IR, FT-Raman spectra and ab initio HF, DFT vibrational analysis of P-methyl acetanilide. Journal of Applied Sciences Research 2009, 11, 1977-1987.

43. El-Mansy, M.A.; Yahia, I. Spectroscopic notes of Methyl Red (MR) dye. Spectrochimica Acta Part A: Molecular Biomolecular Spectroscopy 2014, 130, 59-63, https://doi.org/10.1016/j.saa.2014.03.113.

44. El-Mansy, M.A.; Ismail, M. On the spectroscopic analyses of 3-(4-Hydroxy-1-methyl-2-oxo-1, 2-dihydroquinolin-3-yl)-2-nitro-3-oxo-propionic acid (HMQNP). Spectrochimica Acta Part A: Molecular Biomolecular Spectroscopy 2015, 135, 704-709, https://doi.org/10.1016/j.saa.2014.07.033.

45. El-Mansy, M.A.; El-Nahass, M. On the spectroscopic analyses of Perylene-66. Spectrochimica Acta Part A: Molecular Biomolecular Spectroscopy 2014, 130, 568-573, https://doi.org/10.1016/j.saa.2014.03.118.

46. El-Mansy, M.A.; Mostafa, M.; Ibrahim, M. Synthesis, FT-IR, Bandgap Offset, Polarizability and Hyperpolarizability of 3,4-diamino-6-ehtyl-6H-pyrano[3,2- c]quinoline-2,5-dione (DAPQ) and Cu-2DAPQ as a Promising. Biointerface Research in Applied Chemistry 2021, 11, $8538 \quad-8549$, https://doi.org/10.33263/BRIAC111.85388549.

47. El-Mansy, M.A.; Ismail, M. Structural, conformational, optical, and nonlinear optical behavior of ethyl (6ethyl 5, 6-dihydro 4, 5-dioxo $4 \mathrm{H}$ pyrano [3, 2-c] quinolin-3yl) 2-oxoacetate (EPQOA) : comparative theoretical and experimental studies. Optical and Quantum Electronics 2021, 53, 1-19, https://doi.org/10.1007/s11082-021-02749-7.

48. El-Mansy, M.A.; Suvitha, A.; Narayana, B. Exploring crystal, electronic, optical, and NLO properties of ethyl 4-(3, 4-dimethoxy phenyl)-6-methyl-2-thioxo-1, 2, 3, 4-tetrahydro pyrimidine-5-carboxylate (MTTHPC). Optical and Quantum Electronics 2021, 53, 1-16, https://doi.org/10.1007/s11082-021-03057-w. 
49. Lin, Y.; Rajesh, N.; Raghavan, P.S.; Ramasamy, P.; Huang, Y. Crystal growth of two-component new novel organic NLO crystals. Materials letters 2002, 56, 1074-1077, https://doi.org/10.1016/S0167577X(02)00680-8.

50. Iramain, M.A.; Castillo, M.V.; Davies, L.; Manzur, M.E.; Brandán, S.A. Structural and SQMFF study of potent insecticide 4', 4'-DDT combining the FT-IR and FT-Raman spectra with DFT calculations. Journal of Molecular Structure 2020, 1199, 126964, https://doi.org/10.1016/j.molstruc.2019.126964.

51. Iramain, M.A.; Imbarack, E.; Bongiorno, P.L.; Brandán, S. Characterization of potassium (2-phenylacetyl) trifluoroborate salt by using the UV-Visible, FT-IR and FT-Raman spectra. Journal of Molecular Structure 2020, 1200, 127057, https://doi.org/10.1016/j.molstruc.2019.127057.

52. Selvaraj, S.; Rajkumar, P.; Kesavan, M.; Thirunavukkarasu, K.; Gunasekaran, S.; Devi, N.S.; Kumaresan, S. Spectroscopic and structural investigations on modafinil by FT-IR, FT-Raman, NMR, UV-Vis and DFT methods. Spectrochimica Acta Part A: Molecular Biomolecular Spectroscopy 2020, 224, 117449, https://doi.org/10.1016/j.saa.2019.117449.

53. Iramain, M.A.; Ledesma, A.E.; Imbarack, E.; Bongiorno, P.L.; Brandán, S. Spectroscopic studies on the potassium 1-fluorobenzoyltrifluoroborate salt by using the FT-IR, Raman and UV-Visible spectra and DFT $\begin{array}{llllll}\text { calculations. Journal of } \quad \text { Molecular } & \text { Structure } & \mathbf{2 0 2 0}, & 1204, & 127534,\end{array}$ https://doi.org/10.1016/j.molstruc.2019.127534.

54. El-Mansy, M.A.; Ibrahim, M.; Suvitha, A.; Abdelsalam, H.; Osman. W. Atef, Boosted electronic, optical, and NLO responses of homo P-nanoclusters via conducting polymeric substituents. Computational and Theoretical Chemistry 2021, 113343, https://doi.org/10.1016/j.comptc.2021.113343.

55. Maza, S.; Kijatkin, C.; Bouhidel, Z.; Pillet, S.; Schaniel, D.; Imlau, M.; Guillot, B.; Cherouana, A.; Bendeif, E. Synthesis, structural investigation and NLO properties of three 1, 2, 4-triazole Schiff bases. Journal of Molecular Structure 2020, 128492, https://doi.org/10.1016/j.molstruc.2020.128492.

56. Yadav, S.B.; Sekar, N. Static-and frequency-dependent NLO properties of dithienothiophene and thienothiophene bridges-A computational investigation. Journal of Theoretical and Computational Chemistry 2020, 19, 2050018, https://doi.org/10.1142/S0219633620500182.

57. Mammadova, F.; Ozsinan, S.; Okutan, M.; Dengiz, C. Synthesis, characterization, and theoretical investigation of optical and nonlinear optical (NLO) properties of triazene-based push-pull chromophores. Journal of Molecular Structure 2020, 1220, 128726, https://doi.org/10.1016/j.molstruc.2020.128726.

58. El-Mansy, M.A.; Suvitha, A.; Osman. W.; Khaled, F. Exploring the electronic and optical absorption properties for homo-and hetero-pyrrole-graphene quantum dots. Journal of Computational Electronics 2021, 1-16, https://doi.org/10.1007/s10825-021-01773-w.

59. El-Mansy, M.A.; Osman. W.; Abdelsalam, H. The electronic and optical absorption properties of pristine, homo and hetero Bi-nanoclusters. Chemical Physics 2021, 544, 111113, https://doi.org/10.1016/j.chemphys.2021.111113.

60. Saad, H.A.; Youssef, M.M.; Mosselhi, M. Microwave assisted synthesis of some new fused 1, 2, 4-triazines bearing thiophene moieties with expected pharmacological activity. Molecules 2011, 16, 4937-4957, https://doi.org/10.3390/molecules16064937.

61. Diab, H.M.; Hassan, W.M.; Abdelhamid, I.A.; Elwahy, A.H. Experimental and theoretical study on the regioselective synthesis and reaction of some bis-and poly (3-mercapto-1, 2, 4-triazin-5 (4H)-one) derivatives. Journal of Molecular Structure 2019, $1197, \quad$ 244-261, https://doi.org/10.1016/j.molstruc.2019.07.047.

62. Farag, A.; Halim, S.A.; Roushdy, N.; Badran, A.-S.; Ibrahim, M. Facile synthesis and photodetection characteristics of novel nanostructured triazinyliminomethylpyrano [3, 2-c] quinoline-based hybrid heterojunction. Journal of Molecular Structure $\quad \mathbf{2 0 2 0}, \quad 1221, \quad 128868$, https://doi.org/10.1016/j.molstruc.2020.128868.

63. Fatma, S.; Bishnoi, A.; Verma, A. Synthesis, spectral analysis (FT-IR, 1H NMR, 13C NMR and UV-visible) and quantum chemical studies on molecular geometry, NBO, NLO, chemical reactivity and thermodynamic properties of novel 2-amino-4-(4-(dimethylamino) phenyl)-5-oxo-6-phenyl-5, 6-dihydro-4H-pyrano [3, 2-c] quinoline-3-carbonitrile. Journal of Molecular Structure 2015, 1095, 112-124, https://doi.org/10.1016/j.molstruc.2015.04.026.

64. Shabir, G.; Saeed, A.; Qasim, M.; Bolte, M.; Hökelek, T.; Erben, M. On the planarity of the cyclobutane ring in the crystal of dimethyl 2, 4-bis (3, 4-dimethoxyphenyl) cyclobutane-1, 3-dicarboxylate: a natural bond orbital and Hirshfeld surface analysis study. New Journal of Chemistry 2020, 44, 15515-15525, https://doi.org/10.1039/D0NJ02739A . 
65. Shiroudi, A.; Safaei, Z.; Kazeminejad, Z.; Repo, E.; Pourshamsian, K. DFT study on tautomerism and natural bond orbital analysis of 4-substituted 1, 2, 4-triazole and its derivatives: solvation and substituent effects. Journal of Molecular Modeling 2020, 26, 1-11, https://doi.org/10.1007/s00894-020-4316-9.

66. Kia, R.; Kalaghchi, A. Structural, Non-Covalent Interaction, and Natural Bond Orbital Studies on BromidoTricarbonyl Rhenium (I) Complexes Bearing Alkyl-Substituted 1, 4-Diazabutadiene (DAB) Ligands. Crystals 2020, 10, 267, https://doi.org/10.3390/cryst10040267. 